Florida International University FIU Digital Commons

\title{
Waveguide microgripper for identification, sensing and manipulation
}

Amit G. Bhanushali

Florida International University

DOI: $10.25148 /$ etd.FI14051139

Follow this and additional works at: https://digitalcommons.fiu.edu/etd

Part of the Electrical and Computer Engineering Commons

\section{Recommended Citation}

Bhanushali, Amit G., "Waveguide microgripper for identification, sensing and manipulation" (2009). FIU Electronic Theses and Dissertations. 1670.

https://digitalcommons.fiu.edu/etd/1670

This work is brought to you for free and open access by the University Graduate School at FIU Digital Commons. It has been accepted for inclusion in FIU Electronic Theses and Dissertations by an authorized administrator of FIU Digital Commons. For more information, please contact dcc@fiu.edu. 
FLORIDA INTERNATIONAL UNIVERSITY

Miami, Florida

WAVEGUIDE MICROGRIPPER FOR IDENTIFICATION, SENSING AND

MANIPULATION

A thesis submitted in partial fulfillment of the

requirements for the degree of

MASTER OF SCIENCE

in

ELECTRICAL ENGINEERING

by

Amit G. Bhanushali 
To: Dean Amir Mirmiran

College of Engineering and Computing

This thesis, written by Amit G. Bhanushali, and entitled Waveguide Microgripper for Identification, Sensing and Manipulation, having been approved in respect to style and intellectual content, is referred to you for judgment.

We have read this thesis and recommend that it be approved.

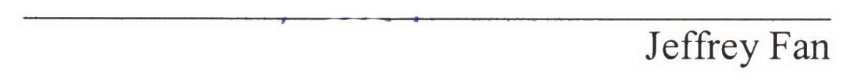

Chunlei Wang

Roberto Panepucci, Major Professor

Date of Defense: March 26, 2009

The thesis of Amit G. Bhanushali is approved.

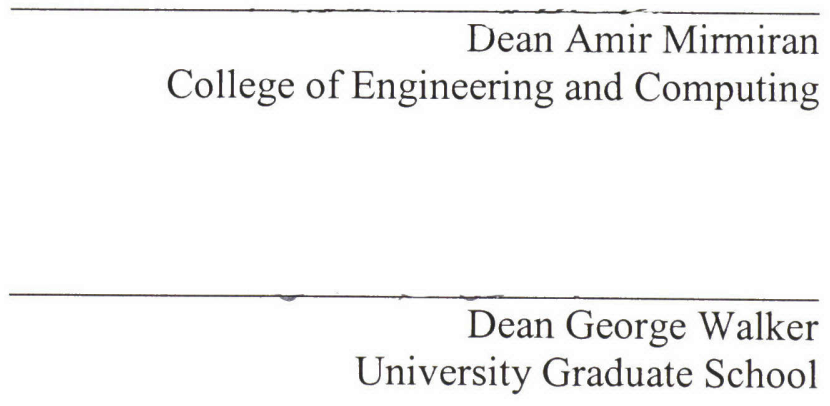

Florida International University, 2009 


\section{DEDICATION}

I dedicate this dissertation to my dear parents Mr Govind Bhanushali and Mrs Vimla Bhanushali, brother Mayur and sister-in-law Manisha for all their love, support and encouragement. 


\section{ACKNOWLEDGMENTS}

I would like to thank Dr. Roberto R. Panepucci for providing me with the opportunity of working on this research and helping me with his knowledge to conduct this research. In addition, I would like to thank Dr. Jeffrey Fan, and Dr. Chunlei Wang for providing valuable feedback and being a part of my committee.

I would like to thank Jose Martinez without whom this thesis would not exist in the first place. His previous work inspired me to take the microgripper project further. Special thanks to Tao Liu for providing his expertise and guiding me through most of the work done in this project. I would also like to thank Xuan Wang, German Vargas, Cristina Rodriguez, Brian Ryan and Kahlil Smith who all contributed in their own way in helping me conducting this research. Last but not the least; I would like to thank all the members of Nanophotonics group for all their support. 


\section{ABSTRACT OF THE THESIS \\ WAVEGUIDE MICROGRIPPER FOR IDENTIFICATION, SENSING AND \\ MANIPULATION \\ by}

Amit G. Bhanushali

Florida International University, 2009

Miami, Florida

Professor Roberto Panepucci, Major Professor

A Waveguide Microgripper utilizes flexible optical waveguides as gripping arms, which provide the physical means for grasping a microobject, while simultaneously enabling light to be delivered and collected. This unique capability allows extensive optical characterization of the structure being held such as transmission, reflection or fluorescence. One of the simplest capabilities of the waveguide microgripper is to be able to detect the presence of a microobject between the microgripper facets by monitoring the transmitted intensity of light coupled through the facets. The intensity of coupled light is expected to drop when there is an object obstructing the path of light. The optical sensing and characterization function of the microgripper is a strong function of the optical power incident on the structure of interest. Hence it is important to understand the factors affecting the power distribution across the facet. The microgripper is also capable of detecting the fluorescence. This capability of microgripper is expected to have applications in medical, bio-medical and related fields. 


\section{TABLE OF CONTENTS}

1 INTRODUCTION

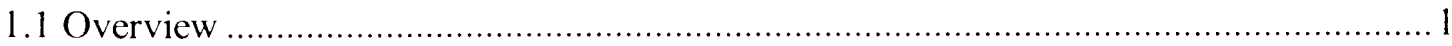

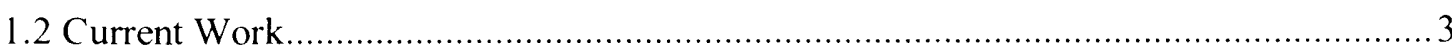

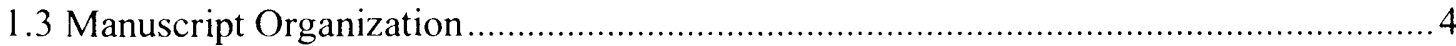

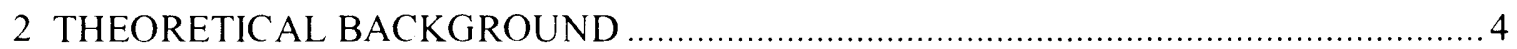

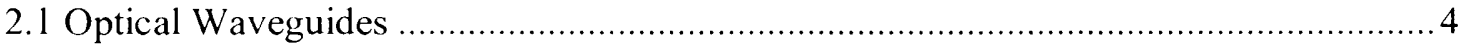

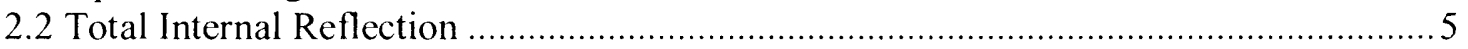

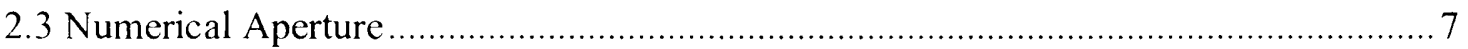

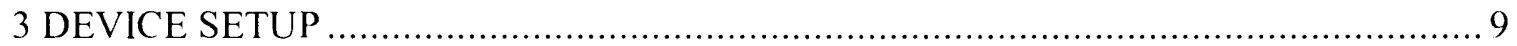

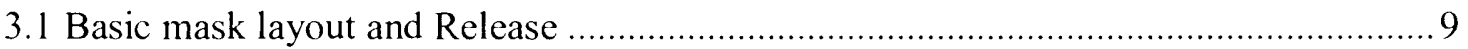

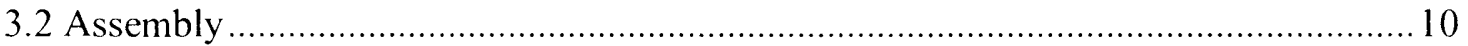

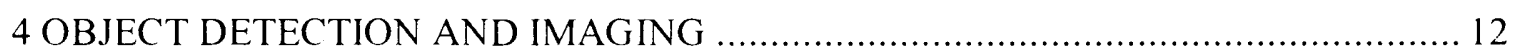

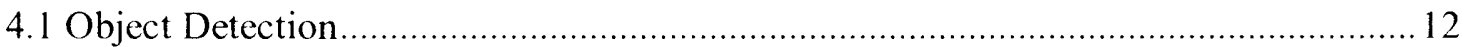

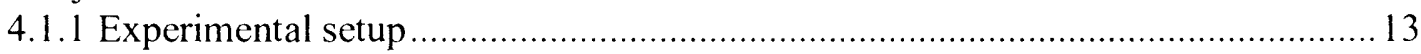

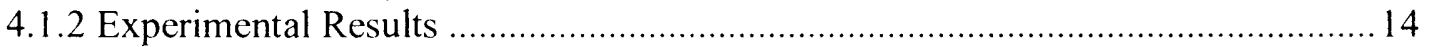

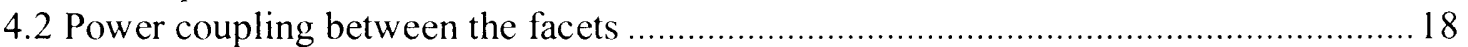

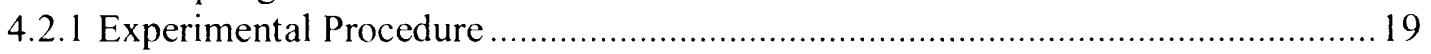

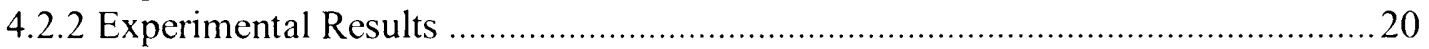

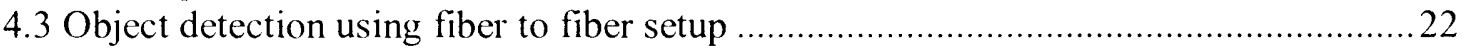

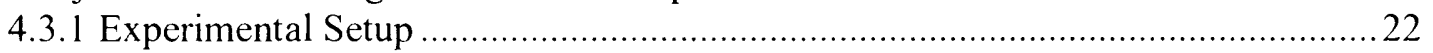

4.3.2 Procedure

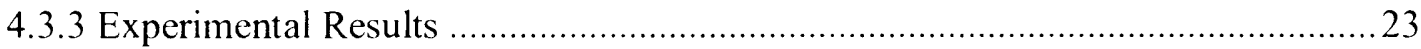

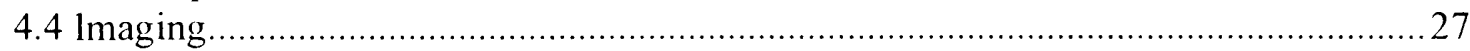

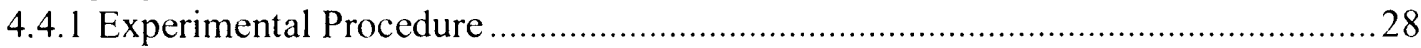

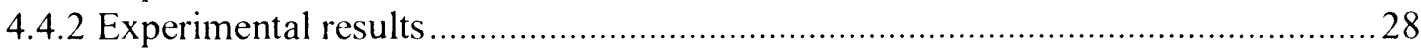

5. FLUORESCENCE DETECTION

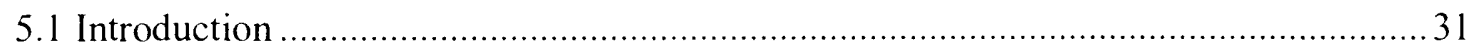

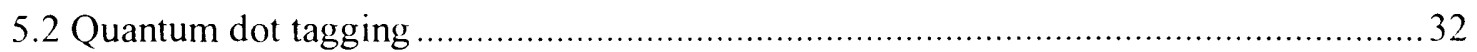

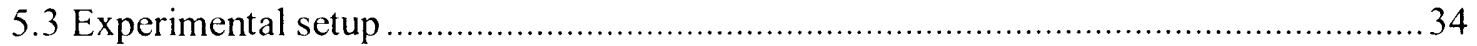

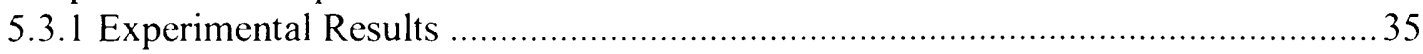

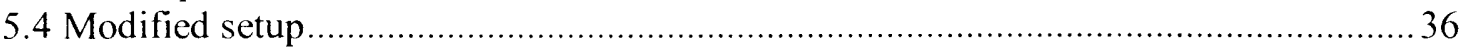

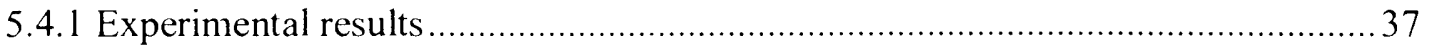

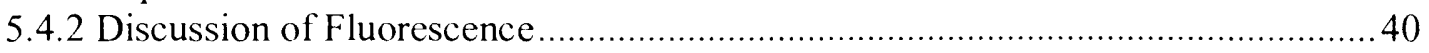

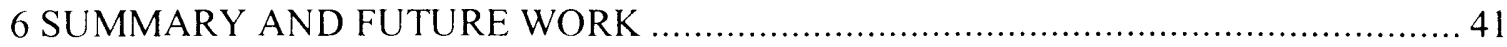

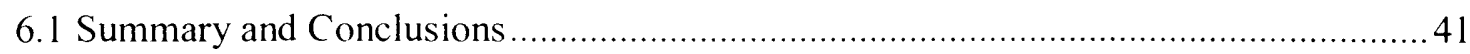

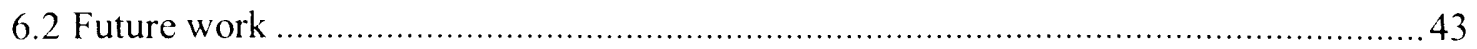

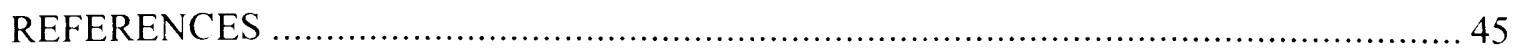




\section{LIST OF FIGURES}

Figure 1: The figure shows the guiding of light through an optical fiber[19] 4

Figure 2: The figure shows the phenomenon of Total Internal Reflection (TIR) when light travels from high refractive index material to low refractive index material [19]

Figure 3: Figure shows the maximum acceptable angle condition to couple the light into an optical fiber[19]

Figure 4: Two rays entering a dielectric waveguide. Ray A enters at an angle $\alpha>\alpha \max$ which causes $\theta<\theta \mathrm{c}$ at the cladding, and consequently allowing ray $A$ to be refracted into the cladding and eventually to escape the waveguide. Ray B enters the waveguide at an an angle $\alpha<\alpha_{\max }$ which causes $\theta>\theta_{c}$ at the cladding, causing total internal reflection [22]. 8

Figure 5: Layout of frame supporting microgrippers in their final design form, with elongated gripping arms to be bonded to optical fibers.[18]

Figure 6: SEM micrograph of released main frame supporting MOEMS microgrippers[18]

Figure 7: Assembly process sequence. 1) Shaft bonding to backbone, 2) first fiber alignment-bonding process, 3) second fiber is bonded with the same procedure, 4) Microgripper is detached from the main frame[18].

Figure 8: Optical micrograph of fiber-coupled $532 \mathrm{~nm}$ green light illuminating the waveguide microgripper (a) knife-edge approaching gap; (b) $20 \mu \mathrm{m}$ thick aluminum slab fully inserted in gap. Notice the significant reduction of light in the output arm and the stronger illumination left arm originated from the reflection of aluminum thin film[24].

Figure 9: Variation in power coupled as a knife-edge is inserted in the gap. Curves are plotted for 4 different gap sizes corresponding to actuator voltages of $0 \mathrm{~V}, 40 \mathrm{~V}, 80 \mathrm{~V}$ and $120 \mathrm{~V}$

Figure 10: Variation in power coupled as a knife-edge is inserted in the gap. Curves are plotted for 4 different gap sizes corresponding to actuator voltages of $0 \mathrm{~V}, 40 \mathrm{~V}, 80 \mathrm{~V}$ and $120 \mathrm{~V}$.....

Figure 11: The figure shows the comparison between the simulation and the actual experiment for the bending losses in the waveguide[18]. 
Figure 12: Transmitted power vs Voltage applied(leading to opening of arms)

for green laser

Figure 13: Transmitted power vs Voltage applied(leading to opening of arms) for blue LED

Figure 14: Layout of a tip of $125 \mu \mathrm{m}$ thick multimode fiber with $62.5 \mu \mathrm{m}$ core diameter.

Figure 15: The graph shows the variation in power coupled as a knife-edge is inserted in the gap for a blue LED light source

Figure 16: The graph shows the variation in power coupled as a knife-edge is inserted in the gap for a green laser light source

Figure 17: The grayscale image of fiber tip illuminated by green laser and the curves for output power vs knife position from two different directions. 26

Figure 18: Schematic of imaging a microgripper facet by a CCD camera. 27

Figure 19: The figure shows the image of the $50 \mu \mathrm{m}$ microgripper facet with white light coming out of it.

Figure 20: The series of images show the facet of microgripper captured by CCD for different positions of input fiber like rotation, pressing and twisting. The change in mode distribution can be clearly seen.

Figure 21: Image a shows the multimode fiber tip illuminated by green laser. Image $b$ shows the same fiber but with perturbation. Image $c$ shows the difference between images $a$ and $b$.

Figure 22: Image a shows the multimode fiber tip illuminated by blue LED. Image $b$ shows the same fiber but with perturbation. Image $c$ shows the difference between images $a$ and $b$. 30

Figure 23: Mechanism of fluorescence emission. 31

Figure 24: Fluorescence detected from SU-8 microparts tagged with QDs with emission at 555, 599, and $612 \mathrm{~nm}$ with an integration time of $500 \mathrm{~ms}$ [18].

Figure 25: Schematic setup for detecting the intensity of fluorescence emission from the quantum dot tagged SU8 films.

Figure 26: Curve representing the relation between Fluorescence intensity and quantum dot concentration. 
Figure 27: Schematic setup of fluorescence detection from a quantum dot tagged SU 8 film.

Figure 28: Schematic of fiber to fiber setup for fluorescence detection of SU8 film

Figure 29: Modified setup to detect the fluorescence from the edges of the SU8 films. Setup on the left is for green laser light source and adjacent setup is for UV light source.

Figure 30: The graph shows the spectrum obtained for fluorescence emitted by $600 \mathrm{~nm}$ Quantum dot doped SU8 films of 4 different concentrations illuminated by UV light source

Figure 31: The graph shows the spectrum obtained for fluorescence emitted by $600 \mathrm{~nm}$ Quantum dot doped SU8 films of 4 different concentrations illuminated by green laser light source. 38

Figure 32: The figure shows the normalized curves for fluorescence intensity measured for highest concentration 600nm Quantum dot tagged films for UV and laser source.

Figure 33: (a)Absorption and photoluminescence spectrum of a CdSe nanocrystal[26], (b)Absorption of white-light CdSe nanocrystals in solution (dashed line), along with emission in solution (solid line)using excitation at $367 \mathrm{~nm}$ [modified from 27] 


\section{INTRODUCTION}

\subsection{Overview}

Microgrippers are promising tools used as end-effectors for systems that can handle and manipulate micro and nano scaled objects with application in various field of science and industry. In the MEMS area, there are many applications where microgrippers have reached an increasing importance. The applications of microgripper structures range from micro manipulation of micro particles, micro components and even cells to assembling and medical applications, and already have a serious impact on present and future technologies[1]. Microgrippers manufactured of polymeric materials offer the advantage of a large displacement and a gentle handling force that can be ideal particularly for specific bioparticle manipulation, as cells. Polymers offer a much lower Young's modulus, and thus much lower actuation and handling forces [2,3]. For example, SU-8 has a Young's modulus in the range of $4.02 \mathrm{GPa}-4.95 \pm 0.42 \mathrm{GPa}[4,5]$ and it was intensively used as structural material for microgrippers in the recent years. Because of its biocompatible properties, SU-8 polymer can be used in a great variety of bio-MEMS applications [6,7]. Experiments that enable the investigation of how individual cells perform their specialised functions, and how they interact between each other, are of crucial importance for the progress of biology and medicine. When studying complex interactions between and inside cells, it is often necessary to hold, sort and transport biological samples in dry or aqueous environments. Current bio-manipulation techniques and tools including optical tweezers, electro kinetic forces, magnetic tweezers, acoustic traps, hydrodynamic flows and pipettes are powerful for particular micro-applications. 
However, these require expensive experimental set-ups and lack the flexibility and ease of use offered by mechanical-end effectors which are in direct contact with the sample but which do not interfere with it either optically or electrically[8]. In recent years, a variety of microgrippers have been developed for the manipulation of micro-sized objects. Different mechanisms of actuation have been used for microgripper applications such as piezoelectric by Carrozza [9], electrostatic by Kim [10], Volland [11], Wierzbicki [12], Shape Memory Alloy (SMA) by Roch [3], or electrothermal by Nguyen [2], Du [13], Chronis[9] and Ivanova [14]. Other important feature that can be integrated in a microgripper is feedback. Feedback mechanism in a microgripper can provide different types of information about the micro object under study. Not many microgripper have been developed with an integrated feedback. The types of feedback implemented in microgrippers are force sensing $[9,15,16]$ and binary optical feedback [17]. Due to limited feedback implementation so far, it is important to integrate a feedback mechanism which would allow the analysis of physical, mechanical, optical and chemical information of the micro object under study. A microgripper with integrated optical feedback has been developed entirely at Florida International University nanofabrication facility by Jose A. Martinez [18]. The device consists of flexible optical waveguides which can act as the gripping arms for grasping a microobject as well as enable the delivery and collection of light. This unique quality allows extensive optical characterization of the microobject under study. The optical feedback helps determine if there is any object or not between the gripping arms. The ability of MOEMS microgripper to simultaneously grasp the microobject and provide optical feedback makes this device a very useful tool in biology and medicine applications. A piezo 
electric actuation mechanism is used which eliminates the need of electrical signal in the vicinity of microgripper, thus making the device very bio-compatible. Fluorescence measurement and microobject detection are some other applications of this device.

\subsection{Current Work}

Single cell manipulation and quantum dot fluorescence detection has been successfully implemented previously by Martinez et al. [18]. The current work focuses mainly on detection of an object blocking the gap between gripping arms and imaging of the microgripper facet. The object detection was demonstrated by inserting a $20 \mu \mathrm{m}$ Aluminum slab in the gap between the arms and monitoring the output power. From the optical feedback received, it can be ascertained if the object is within the gap or not. Also from the amount of power drop, the approximate position of the microobject can be determined. The other important study performed on the device was the imaging of the microgripper facet. This was done by taking apart one half of the microgripper and placing a $\mathrm{CCD}$ in front of the retained facet in order to image it. The retained half of the microgripper was illuminated by a $532 \mathrm{~nm}$ green laser and the illumination across the microgripper facet was studied. Both object detection and imaging were also demonstrated using a fiber to fiber setup. The setup resembles the microgripper structure very closely and hence it was interesting to compare the results. The fluorescence detection experiment that was performed previously by Martinez et al. [18] was repeated for lower concentration to check the sensitivity of the microgripper. 


\subsection{Manuscript Organization}

The manuscript is divided into six chapters including the current one. Chapter 2 provides the necessary background information for the performed study which is the optical waveguides and their properties. Chapter 3 provides the procedure for basic device setup which includes the mask layout, release and assembly of the microgripper. Chapter 4 details the object detection ability of the microgripper. Later in the chapter, the imaging of microgripper facet is discussed. Chapter 5 discusses the fluorescence detection ability of the microgripper. It discusses the previously performed experiments and the experiments performed in this work to test the sensitivity of the microgripper. Chapter 6 presents a summary of the research work performed followed by conclusions and suggested future work.

\section{THEORETICAL BACKGROUND}

\subsection{Optical Waveguides}

An optical waveguide is a medium that is capable of guiding light. The optical fibers used are composed of a dielectric material (core) surrounded by a second dielectric material (cladding) with lower index of refraction.

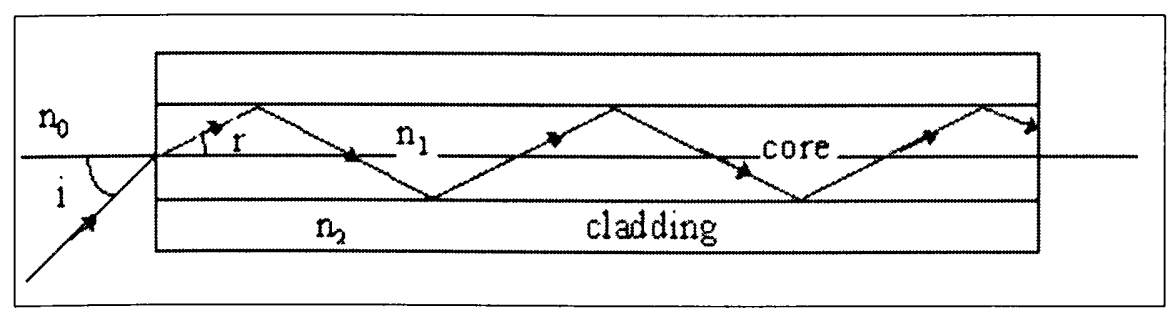

Figure 1: The figure shows the guiding of light through an optical fiber[19] 
This structure is capable of confining electromagnetic energy in the form of light, and it guides it in the direction of its axis of symmetry. The microgrippers studied in this work make use of optical fibers for transmitting light through them. Hence it is important to describe some of the very important concepts of waveguides like Total Internal Reflection (TIR) and Numerical Aperture(NA)

\subsection{Total Internal Reflection}

When light propagates in free space it can be treated as a ray, and depending on the medium that it goes through, it changes direction due to refraction. When a light ray strikes a smooth interface between two transparent media at an angle, it is refracted [20]. Consequently the angle of the resulted ray will be different than the one that struck on the other side. Different materials have different properties that cause this refraction, and that property is called the index of refraction (n). The $n$ value describes the sharpness of the refraction at the interface. One of the basic laws that describe the behavior of light as it penetrates from one medium to the other is Snell's law[21]. For understanding the concept of light confinement in a medium, consider the case of a ray of light travelling in a medium of refractive index $n_{l}$, and incident to the the boundary with second medium of refractive index $n_{2}$. Consider $\theta_{1}$ as angle of incidence and $\theta_{2}$ as angle of transmission (also called refraction). Snell's law[21] shows the relation between two mediums using the indices of refraction, the incident angle and the refracted angle is such way that

$$
n_{1} \sin \theta_{1}=n_{2} \sin \theta_{2}
$$


which can also be expressed as

$$
\frac{n_{1}}{n_{2}}=\frac{\sin \theta_{2}}{\sin \theta_{1}}
$$

From equation 2.2 it can be seen that when light propagates from a medium with higher index of refraction to a medium with lower index, $\theta_{2}$ will be greater than $\theta_{1}$ and when light propagates from lower to higher index mediums, $\theta_{2}$ will be smaller than $\theta_{1}$. As a rule of thumb, light will always be deflected towards the higher index medium

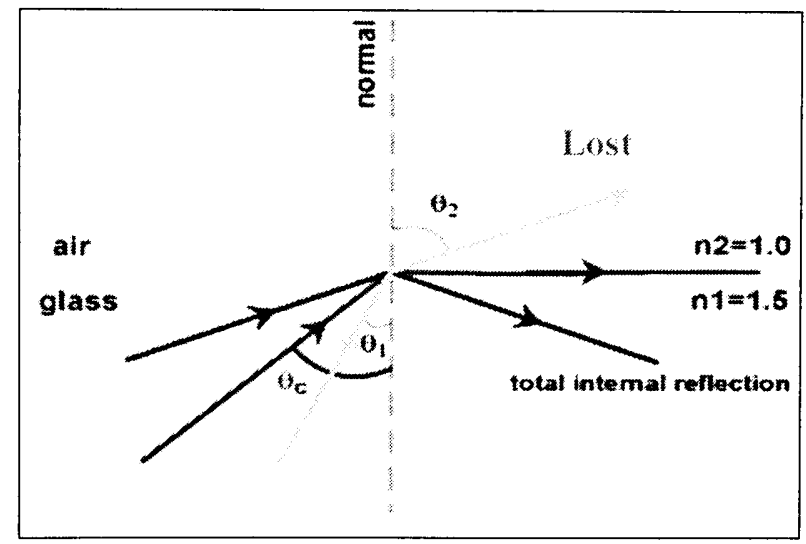

Figure 2: The figure shows the phenomenon of Total Internal Reflection (TIR) when light travels from high refractive index material to low refractive index material [19]

When light crosses a boundary between materials with different refractive indices, the light beam will be partially refracted at the boundary surface, and partially reflected. However, if the angle of incidence is greater than the critical angle $\theta_{c}$, then the light will stop crossing the boundary altogether and instead be totally reflected back internally.

$$
\sin \theta_{c}=\frac{n_{2}}{n_{1}}
$$


This can only occur where light travels from a medium with a higher refractive index to one with a lower refractive index.

\subsection{Numerical Aperture}

The ability of a waveguide to collect light incident onto its input facet is determined by its numerical aperture $(N A)$. In analogy to the $N A$ of a lens, it is an indication of the half angle of light that is 'focused' into the guided modes.

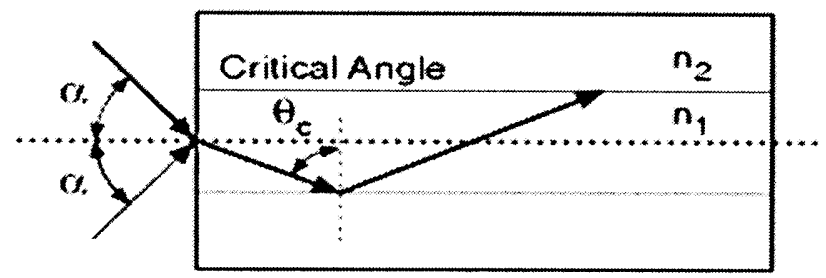

Figure 3: Figure shows the maximum acceptable angle condition to couple the light into an optical fiber[19]

From Snell's law[21], and the total internal reflection previously discussed, the following relation is obtained:

$$
n_{0} \sin \left(\alpha_{\max }\right)=n_{1} \sin \left(\theta_{c}\right)=\sqrt{n_{1}^{2}-n_{2}^{2}}
$$

Where $n_{0}$ is the index of refraction of the medium in front of the facet, $n_{l}$ is the index of refraction of the core, $n_{2}$ is the refractive index of the cladding, and $\alpha_{\max }$ is the maximum acceptance angle. The angle of the cone formed by the maximum acceptance angle is given by $2 \alpha_{\max }$ and it is referred to as total acceptance angle. The numerical aperture $N A$ of the waveguide can be expressed as:

$$
N A=n_{0} \cdot \sin \left(\alpha_{\text {max }}\right)=\sqrt{n_{1}^{2}-n_{2}^{2}}
$$


The previous discussion can be illustrated with figure 4, which depicts two rays entering a dielectric waveguide. Ray $A$ does not propagate along the waveguide, and ray $B$ does propagate along the axis of symmetry of the waveguide. Ray $A$ arrives to the facet at an incident angle greater than $\alpha_{\max }$, which causes its transmitted fraction to travel inside the core of the waveguide for a short distance, until it reaches the cladding at a smaller angle than $\theta_{c}$, not sufficient for TIR, and consequently propagating into the cladding and finally escaping the waveguide. Ray $B$ arrives to the facet at an incident angle smaller than $\alpha_{\max }$, which causes its transmitted fraction to travel inside the core, and to arrive to the cladding at an angle greater than $\theta_{c}$, sufficient to cause TIR, and therefore reflecting the ray back towards the center of the waveguide, and repeating the process every time the cladding is met.

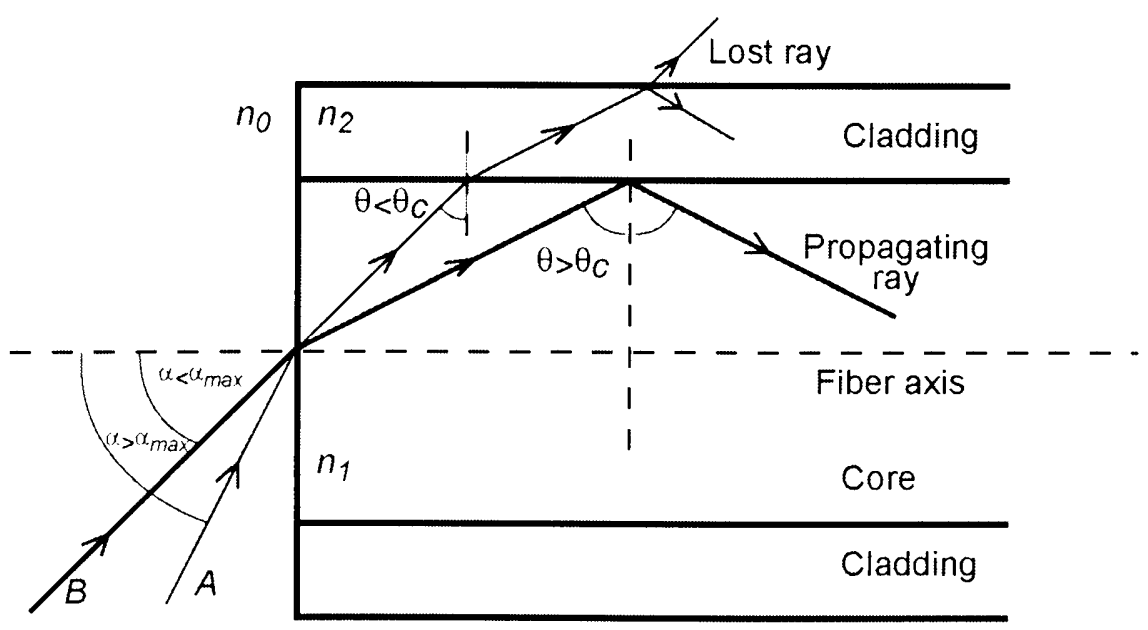

Figure 4: Two rays entering a dielectric waveguide. Ray $A$ enters at an angle $\alpha>\alpha \max$ which causes $\theta$ $<\theta \mathrm{c}$ at the cladding, and consequently allowing ray $\mathrm{A}$ to be refracted into the cladding and eventually to escape the waveguide. Ray $B$ enters the waveguide at an an angle $\alpha<\alpha_{\max }$ which causes $\theta>\theta_{c}$ at the cladding, causing total internal reflection [22]. 


\subsection{Basic mask layout and Release}

The mask was completely designed at FIU nanofabrication facility by Jose Martinez[18]. The basic mask layout comprised of frames supporting microgrippers with arm widths 10, 20, 30, 40, 50 and 100 microns. Every frame had a pair of each of these sizes. Each microgripper in a frame is attached by thin stubs strong enough to hold microgripper in place during the microfabrication process but weak enough to separate the microgripper during the assembly.

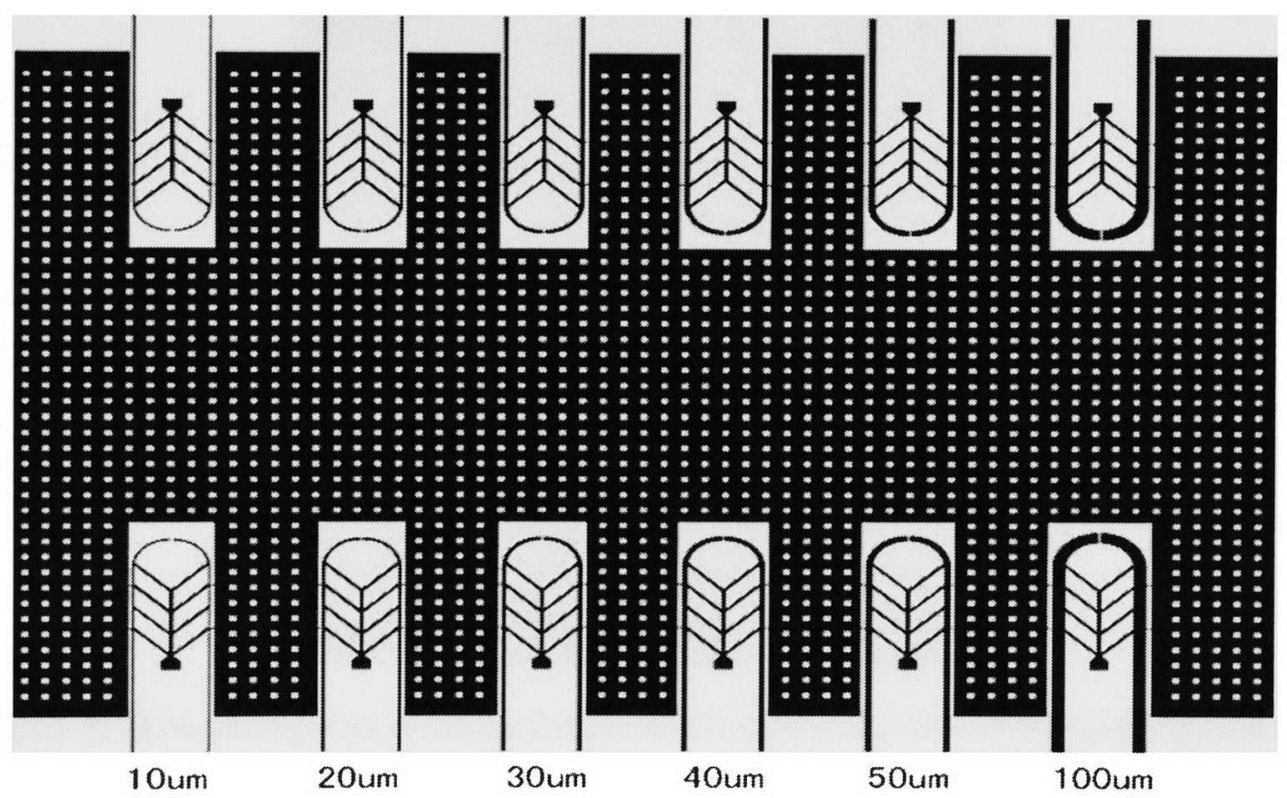

Figure 5: Layout of frame supporting microgrippers in their final design form, with elongated gripping arms to be bonded to optical fibers.[18]

Two types of frames were designed, one having a set of microgrippers with short arms and the other with elongated arms microgrippers as shown above. The short arm grippers can be used only for mechanical operations and characterization purposes, and the ones with elongated arms are meant to be bonded to optical fibers to enable transmission of 
light. The microgrippers with elongated arms have been used for all the experiments discussed in this work. To release the frame from substrate, the complete structure is immersed in a buffers Hydrofluoric acid (buffered oxide etch) for approximately 10 mins. The SU8 frame consisting of microgrippers comes afloat on the acid surface which can then be picked up by a tweezer. The structure is cleaned with deionized (DI) water and placed it on a absorbant tissue. At this point the microgrippers are ready for assembly. Figure below shows SEM image of a released frame.

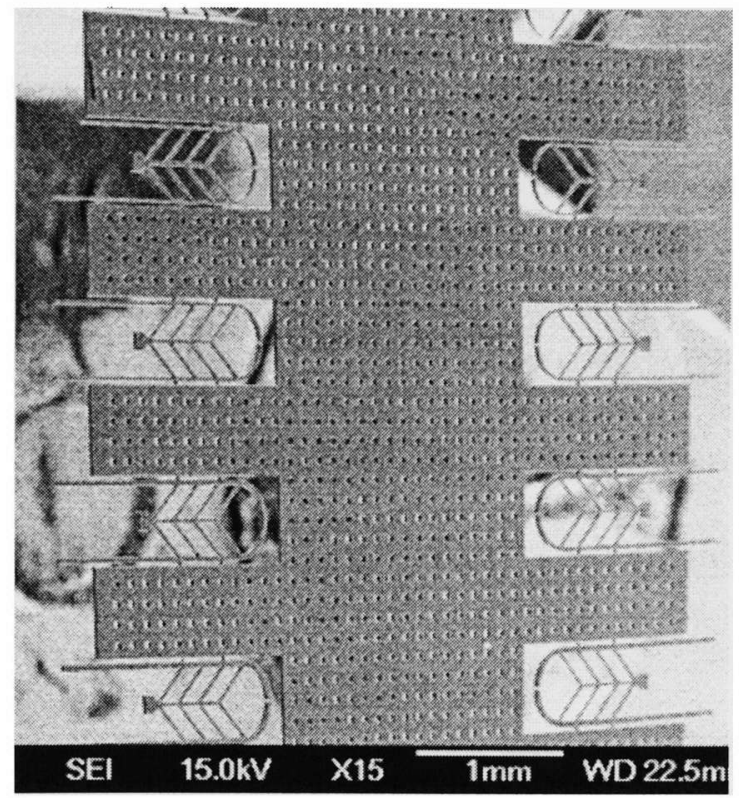

Figure 6: SEM micrograph of released main frame supporting MOEMS microgrippers[18]

\subsection{Assembly}

The microgripper was assembled using the steps developed previously by Jose Martinez[18]. To begin with, a frame consisting of microgrippers is placed on a XYZ stage. A small length bare optical fiber(shaft) is attached to the piezo electric actuator which will lead the microgripper to open when a voltage is applied. The shaft is passed 
through a $150 \mu \mathrm{m}$ diameter hollow needle. The tip of the shaft is coated with a blob of UV curable glue. The tip of the shaft is kept far away from the needle to prevent the glue from sticking to needle. The frame of microgripper is moved using the $\mathrm{XYZ}$ stage and the backbone of desired sized microgripper is visually aligned to the UV coated shaft as shown in Fig 7(1). A physical contact is made between the two and the UV glue is cured using an Ultraviolet lamp to establish a strong bond between the shaft and gripper backbone. Once the bond is established, the frame is pulled towards the needle using a manual micrometer screw. At this point, an optical fiber with a small bend that allows it to move parallel to the shaft, is placed on a second $\mathrm{XYZ}$ stage. The tip of the fiber is coated with UV curable glue and it is visually aligned to the waveguide input as shown in Fig 7(2). The $532 \mathrm{~nm}$ green laser light is passed through the input fiber to locate a point where maximum coupling is obtained. The configuration is maintained and the UV glue is cured using the UV lamp. The same procedure is followed to align another fiber to the waveguide output. To determine the best alignment, the other end of the output fiber is connected to a photodetector and the fiber is UV cured at the position where maximum power is obtained. Some extra glue is applied in the area between the fibers and the needle to make the system more robust as shown in Fig 7(3). At this point, the microgripper is ready to be separated from the frame. A gentle pull is enough to release the microgripper from the frame. The final assembled device is shown in Fig 7(4). The microgrippers with arm width $50 \mu \mathrm{m}$ and $100 \mu \mathrm{m}$ were successfully assembled but the ones below $50 \mu \mathrm{m}$ were difficult to assemble. All the experiments performed in this work are with $100 \mu \mathrm{m}$ microgripper except the one that was used for imaging is $50 \mu \mathrm{m}$. 


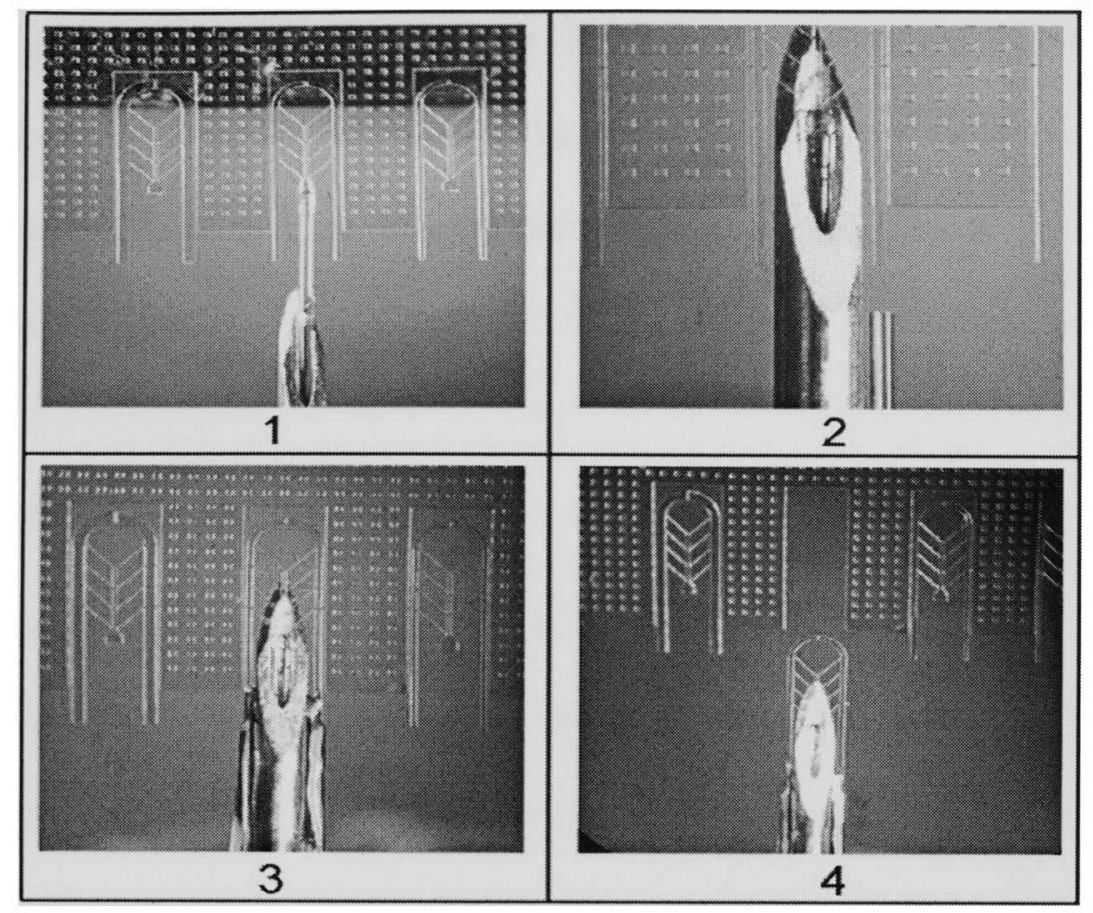

Figure 7: Assembly process sequence. 1) Shaft bonding to backbone, 2) first fiber alignment-bonding process, 3) second fiber is bonded with the same procedure, 4) Microgripper is detached from the main frame[18].

\section{OBJECT DETECTION AND IMAGING}

\subsection{Object Detection}

Novel waveguide microgrippers have many applications in micro-assembly and cell biology as they enable the simultaneous manipulation and optical characterization of micro/nanostructures. The optical sensing and characterization function of the device is a strong function of the optical power incident on the structure of interest, hence it is important to understand the factors affecting the power distribution across the facet. One of the simplest capabilities of the waveguide microgripper is to be able to detect the presence of a microobject between the microgripper facets by monitoring the transmitted 
intensity of light coupled through the facets. The intensity of coupled light is expected to drop when there is an object obstructing the path of light. This would be achieved by simple binary feedback to determine if there is a microstructure or not between the gripping arms[23].

\subsubsection{Experimental setup}

The knife edge method was used to investigate the output optical power. Laser light was coupled from a $128 \mathrm{~mW}, 532 \mathrm{~nm} \mathrm{Nd:YAG} \mathrm{to} \mathrm{the} \mathrm{input} \mathrm{fiber,} \mathrm{while} \mathrm{monitoring}$ the fiber-coupled output power with a photo-detector. The transmission through the arms of the microgripper was measured as the gap between the two arms was blocked by a 20 $\mu \mathrm{m}$ thick aluminum slab (used as knife edge) introduced in the gap. Fig. 8 shows an optical micrograph of the gripper before and after the knife edge was inserted between the two waveguide arms.

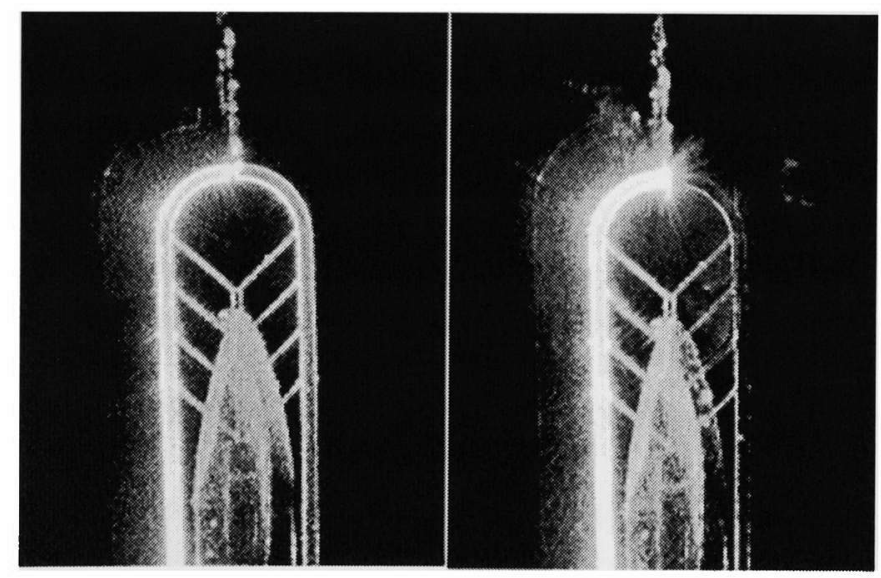

a.

b

Figure 8: Optical micrograph of fiber-coupled $532 \mathrm{~nm}$ green light illuminating the waveguide microgripper (a) knife-edge approaching gap; (b) $20 \mu \mathrm{m}$ thick aluminum slab fully inserted in gap. Notice the significant reduction of light in the output arm and the stronger illumination left arm originated from the reflection of aluminum thin film[24] 
The output power was measured at the right arm for different positions of the knife-edge. Also, the opening of the gripper was changed by varying the voltage applied to the piezoelectric actuator. The starting position of the knife edge was $50 \mu \mathrm{m}$ away from the gripper, and its final position was $50 \mu \mathrm{m}$ past the inner edge of the microgripper. Hence the knife-edge covered the total distance of $200 \mu \mathrm{m}$ including the $100 \mu \mathrm{m}$ of gripper width. The knife edge was moved towards the gripper of $100 \mu \mathrm{m}$ width in steps of $10 \mu \mathrm{m}$. The value of output power was noted at each step. The initial actuator voltage was $0 \mathrm{~V}$ which means that the gripper opening was approximately $16 \mu \mathrm{m}$, as defined by lithography. The actuator voltage was increased in steps of $10 \mathrm{~V}$ up to $120 \mathrm{~V}$. Increasing the voltage leads to a widening of the opening gap of the gripper, so larger objects could be manipulated.

\subsubsection{Experimental Results}

The knife reaches the edge of gripper after $50 \mu \mathrm{m}$ travel, and then it starts entering the gripper from $60 \mu \mathrm{m}$ onwards. At $150 \mu \mathrm{m}$ the knife edge reaches the inside edge of the gripper facet and as we move to $160 \mu \mathrm{m}$, the entire gap is blocked. From the results obtained in figure 9, it can be seen that the output power increases from the initial position until the knife-edge reaches the $50 \mu \mathrm{m}$ position. This increase could be due to reflections from the approaching aluminum film increasing the light coupled to the output waveguide. Also from the pictures it is clear that for the laser source, some amount of light is scattered outside the waveguide. The scattering is more visible around the opening between input and output waveguide. To verify that the slight increase in output power before the knife reaching the edge is due to reflection, the same experiment was 
performed using a $592 \mathrm{~nm}$ Yellow LED light source. The results for yellow LED show that the output power is constant until the knife reaches the outer edge of the gap. After that point the output power decreases linearly. At $150 \mu \mathrm{m}$, the knife-edge completely blocks the gap between two arms of the gripper, and consequently the output power remains constant from $160 \mu \mathrm{m}$ through $200 \mu \mathrm{m}$.

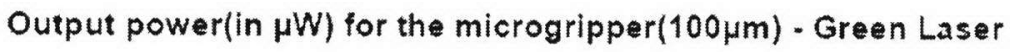

\begin{tabular}{|c|c|c|c|c|c|c|c|c|c|c|c|c|c|}
\hline $\begin{array}{l}\text { Plezo } \\
\text { Voltage }\end{array}$ & ov & $10 \mathrm{~V}$ & $20 \mathrm{~V}$ & $30 \mathrm{~V}$ & $40 \mathrm{~V}$ & $50 \mathrm{~V}$ & $60 \mathrm{~V}$ & $70 \mathrm{~V}$ & $80 \mathrm{~V}$ & $90 \mathrm{~V}$ & $100 \mathrm{~V}$ & $10 \mathrm{~V}$ & $120 \mathrm{~V}$ \\
\hline \multicolumn{14}{|l|}{ Diatance } \\
\hline oum & 3.65 & 3.6 & 3.65 & 3.6 & 3.5 & 3.65 & 3.45 & 3.45 & 3.35 & 3.29 & 3.23 & 3.1 & 3 \\
\hline $10 \mu \mathrm{m}$ & 3.65 & 3.61 & 3.65 & 3.7 & 3.5 & 3.6 & 3.56 & 3.45 & 3.3 & 3.36 & 321 & 3.05 & 3.08 \\
\hline $20 \mu \mathrm{m}$ & 3.63 & 3.65 & 3.7 & 3.5 & 3.61 & 3.6 & 3.5 & 3.53 & 32 & 3.38 & 3.29 & 3 & 3.09 \\
\hline $30 \mu \mathrm{m}$ & 3.6 & 3.69 & 382 & 3.54 & 3.65 & 3.5 & 3.57 & 3.57 & 3.25 & 3.31 & 329 & 2.98 & 2.91 \\
\hline $40 \mu \mathrm{m}$ & 3.7 & 3.75 & 3.85 & 3.8 & 3.65 & 3.55 & 3.61 & 3.61 & 3.3 & 3.32 & 3.3 & 2.95 & 2.96 \\
\hline 50بm & 3.8 & 3.8 & 3.87 & 3.8 & 3.7 & 3.5 & 3.7 & 3.51 & 3.41 & 3.27 & 3.21 & 2.99 & 3.05 \\
\hline $60 \mu \mathrm{m}$ & 3.6 & 3.9 & 3.5 & 3.4 & 3.45 & 3.33 & 3.43 & 3.31 & 32 & 3.28 & 3.1 & 2.94 & 3.03 \\
\hline $70 \mu m$ & 2.81 & 2.87 & 2.95 & 2.8 & 2.9 & 2.9 & 2.99 & 2.9 & 2.69 & 2.71 & 2.75 & 26 & 2.91 \\
\hline $80 \mu m$ & 2.16 & 2.19 & 2.1 & 245 & 2.3 & 2.26 & 2.36 & 2.48 & 2.13 & 2.19 & 2.15 & 2.33 & 2.41 \\
\hline $90 \mu \mathrm{m}$ & 1.71 & 1.69 & 166 & 188 & 1.8 & 176 & 1.92 & 1.83 & 1.7 & 1.55 & 1.7 & 1.76 & 1.92 \\
\hline $100 \mu \mathrm{m}$ & 1.3 & 1.4 & 128 & 1.35 & 1.36 & 1.36 & 1.56 & 1.55 & 1.36 & 1.16 & 1.28 & 1.39 & 1.54 \\
\hline $110 \mu \mathrm{m}$ & 0.95 & 0.87 & 1.04 & 1.07 & 1.02 & 1.03 & 1.05 & 1.15 & 1.01 & 0.98 & 0.96 & 1.06 & 1.16 \\
\hline $120 \mu \mathrm{m}$ & 0.49 & 0.54 & 0.7 & 0.81 & 0.73 & 0.72 & 0.82 & 0.9 & 0.81 & 0.75 & 0.76 & 0.81 & 0.82 \\
\hline $130 \mu \mathrm{m}$ & 0.24 & 0.32 & 0.35 & 0.55 & 0.47 & 0.46 & 0.62 & 0.65 & 0.64 & 0.51 & 0.54 & 0.54 & 0.61 \\
\hline $140 \mu \mathrm{m}$ & 0.083 & 0.085 & 0.203 & 0.38 & 0.2 & 0.25 & 0.42 & 0.46 & 0.41 & 0.34 & 0.33 & 0.36 & 0.38 \\
\hline $150 \mu \mathrm{m}$ & 0.032 & 0.025 & 0.075 & 0.2 & 0.09 & 0.092 & 0.26 & 0.27 & 0.24 & 0.19 & 0.17 & 0.22 & 0.26 \\
\hline $160 \mu \mathrm{m}$ & 0.024 & 0.022 & 0.025 & 0.07 & 0.05 & 0.046 & 0.15 & 0.16 & 0.12 & 0.06 & 0.06 & 0.09 & 0.07 \\
\hline $170 \mu \mathrm{m}$ & 0.022 & 0.02 & 0.023 & 0.03 & 0.035 & 0.035 & 0.06 & 0.07 & 0.05 & 0.033 & 0.028 & 0.031 & 0.03 \\
\hline $180 \mu \mathrm{m}$ & 0.022 & 0.02 & 0.023 & 0.028 & 0.035 & 0.035 & 0.04 & 0.035 & 0,035 & 0.033 & 0.027 & 0.029 & 0.026 \\
\hline $190 \mu \mathrm{m}$ & 0.022 & 0.02 & 0.023 & 0.028 & 0.035 & 0.035 & 0.04 & 0.035 & 0.035 & 0.033 & 0.027 & 0.029 & 0.026 \\
\hline $200 \mu \mathrm{m}$ & 0.022 & 0.02 & 0.023 & 0.028 & 0.035 & 0.035 & 0.04 & 0.035 & 0.035 & 0033 & 0.027 & 0.029 & 0.026 \\
\hline
\end{tabular}

Table 1 - Output power in $\mu \mathrm{W}$ for piezo voltages varying from $0 \mathrm{~V}$ through $120 \mathrm{~V}$ and object distance varying from $0 \mu \mathrm{m}$ to $200 \mu \mathrm{m}$ for green laser. 


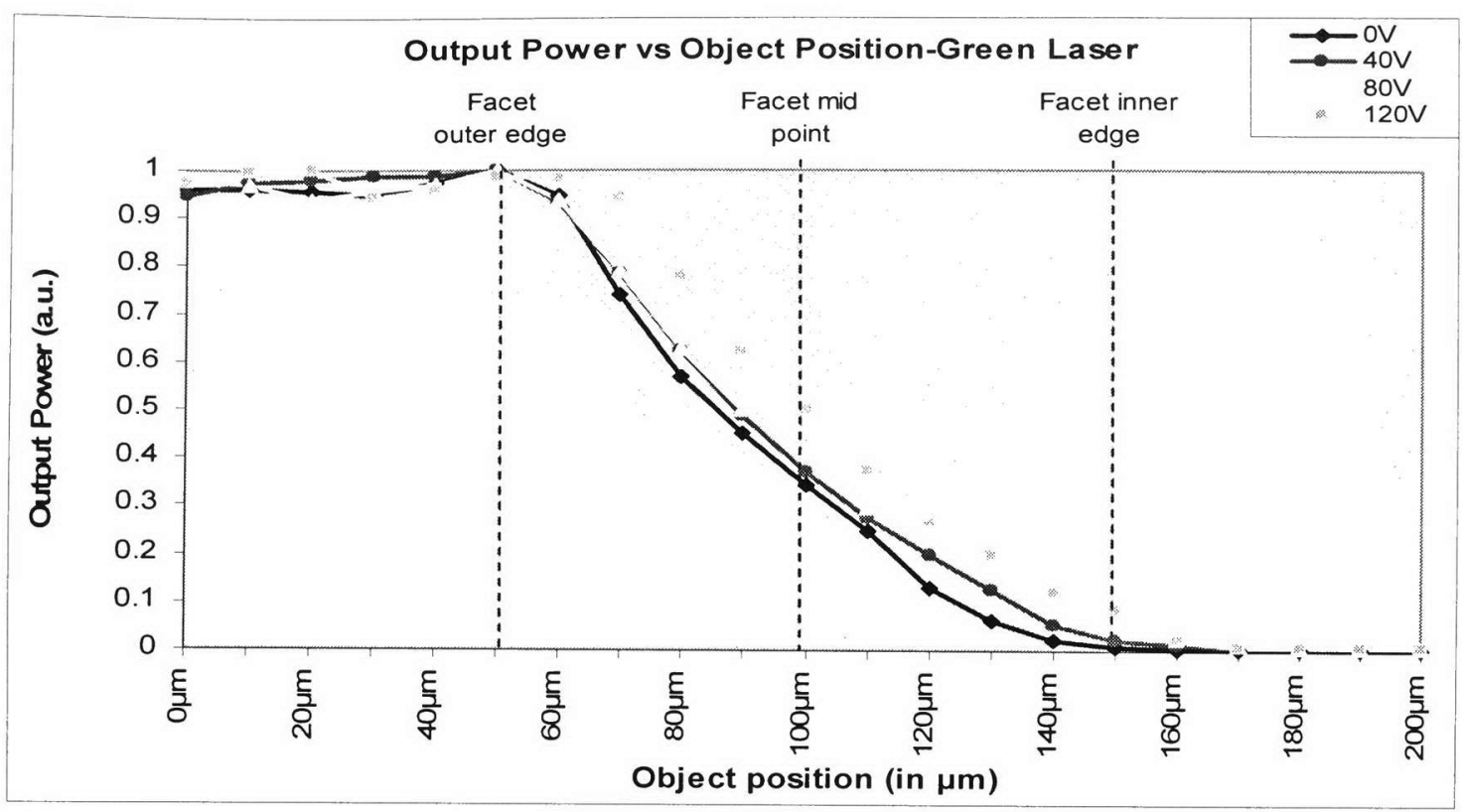

Figure 9: Variation in power coupled as a knife-edge is inserted in the gap. Curves are plotted for 4 different gap sizes corresponding to actuator voltages of $0 \mathrm{~V}, 40 \mathrm{~V}, 80 \mathrm{~V}$ and $120 \mathrm{~V}$.

\begin{tabular}{|c|c|c|c|c|c|c|c|c|c|c|c|c|c|}
\hline \multicolumn{14}{|c|}{ Output power(in nTV) for the microgripper - Yellow LED } \\
\hline $\begin{array}{l}\text { Plezo } \\
\text { Voltage } \\
\end{array}$ & ov & $10 \mathrm{~V}$ & $20 \mathrm{~V}$ & $30 \mathrm{~V}$ & $40 \mathrm{~V}$ & $50 \mathrm{~V}$ & $60 \mathrm{~V}$ & $70 \mathrm{~V}$ & $80 \mathrm{~V}$ & $90 \mathrm{~V}$ & $100 \mathrm{~V}$ & $110 \mathrm{~V}$ & $120 \mathrm{~V}$ \\
\hline \multicolumn{14}{|l|}{ Diatance } \\
\hline oum & 0.47 & 0.46 & 0.44 & 0.44 & 0.44 & 0.43 & 0.41 & 0.41 & 0.4 & 0.37 & 0.37 & 0.36 & 0.36 \\
\hline $10 u m$ & 0.47 & 0.46 & 0.44 & 0.44 & 0.44 & 0.43 & 0.41 & 0.41 & 0.4 & 0.37 & 0.37 & 0.36 & 0.36 \\
\hline $20 \mu \mathrm{m}$ & 0.47 & 0.46 & 0.44 & 0.44 & 0.44 & 0.43 & 0.41 & 0.41 & 0.4 & 0.37 & 0.37 & 0.36 & 0.36 \\
\hline $30 u m$ & 0.47 & 0.46 & 0.44 & 0.44 & 0.44 & 0.43 & 0.41 & 0.41 & 0.4 & 0.37 & 0.37 & 0.36 & 0.36 \\
\hline $40 u m$ & 0.47 & 0.46 & 0.44 & 0,44 & 0.44 & 0.43 & 0.41 & 0.41 & 0.4 & 0.37 & 0.37 & 0.36 & 0.36 \\
\hline 50um & 0.47 & 0.46 & 0.44 & 0.44 & 0.44 & 0.43 & 0.41 & 0.41 & 0.4 & 0.37 & 0.37 & 0.36 & 0.36 \\
\hline 60um & 0.45 & 0.44 & 0.43 & 0.4 & 04 & 0.37 & 0.38 & 0.38 & 0.37 & 0.35 & 0.34 & 0.33 & 0.33 \\
\hline $70 u m$ & 0.41 & 0.39 & 0.38 & 0.33 & 0.31 & 0.32 & 0.32 & 0.32 & 0.31 & 0.28 & 0.29 & 0.28 & 0.28 \\
\hline 80um & 0.3 & 0.31 & 0.3 & 0.25 & 0.24 & 0.25 & 0.25 & 0.25 & 0.23 & 0.22 & 0.22 & 0.21 & 0.22 \\
\hline goum & 0.24 & 0.24 & 021 & 0.19 & 0.18 & 0.19 & 0.19 & 0.19 & 0.18 & 0.18 & 0.16 & 0.16 & 0.16 \\
\hline $100 \mu m$ & 0.10 & 0.19 & 0.1 & 0.14 & 0.13 & 0.14 & 0.15 & 0.15 & 4 & 0. & 0.13 & 0.12 & 0.12 \\
\hline $110 u m$ & 0.14 & 0.13 & 0.13 & 0.1 & 0.1 & 0.11 & 0.11 & 0.11 & 0.11 & 0.09 & 0.09 & 0.08 & 0.09 \\
\hline $120 \mu \mathrm{m}$ & 0.1 & 0.11 & 0.09 & 0.07 & 0.07 & 0.08 & 0.07 & 0.08 & 0.08 & 0.06 & 0.06 & 0.05 & 0.05 \\
\hline $130 u m$ & 0.05 & 0.07 & 0.07 & 0.03 & 0.03 & 0.05 & 0.05 & 0.05 & 0.05 & 0.04 & 0.04 & 0.04 & 0.03 \\
\hline $140 u m$ & 0.02 & 0.03 & 003 & 0.02 & 0.02 & 0.02 & 0.02 & 0.03 & 0.02 & 0.02 & 0.01 & 0.02 & 0.02 \\
\hline $150 \mu \mathrm{m}$ & 0.01 & 0.01 & 001 & 0.01 & 0.01 & 0.01 & 0.01 & 0.01 & 0.01 & 0.01 & 0.01 & 0.01 & 0.01 \\
\hline $160 \mu \mathrm{m}$ & 0.005 & 0.005 & 0.005 & 0.005 & 0.005 & 0.005 & 0.01 & 0.005 & 0.005 & 0.005 & 0.005 & 0.005 & 0.005 \\
\hline $170 \mu \mathrm{m}$ & 0.005 & 0.005 & 0.005 & 0.005 & 0.005 & 0.005 & 0.01 & 0.005 & 0.005 & 0.005 & 0.005 & 0.005 & 0.005 \\
\hline $180 \mu \mathrm{m}$ & 0.005 & 0.005 & 0.005 & 0.005 & 0.005 & 0.005 & 0.01 & 0.005 & 0.005 & 0.005 & 0.005 & 0.005 & 0.005 \\
\hline 190um & 0.005 & 0.005 & 0.005 & 0.005 & 0.005 & 0.005 & 0.01 & 0.005 & 0.005 & 0.005 & 0.005 & 0.005 & 0.005 \\
\hline $200 u m$ & 0.005 & 0.005 & 0.005 & 0.005 & 0.005 & 0.005 & 0.01 & 0.005 & 0.005 & 0.005 & 0.005 & 0.005 & 0.005 \\
\hline
\end{tabular}

Table 2 - Output power in $\mathrm{nW}$ for piezo voltages varying from $0 \mathrm{~V}$ through $120 \mathrm{~V}$ and object distance varying from $0 \mu \mathrm{m}$ to $200 \mu \mathrm{m}$ for yellow LED. 


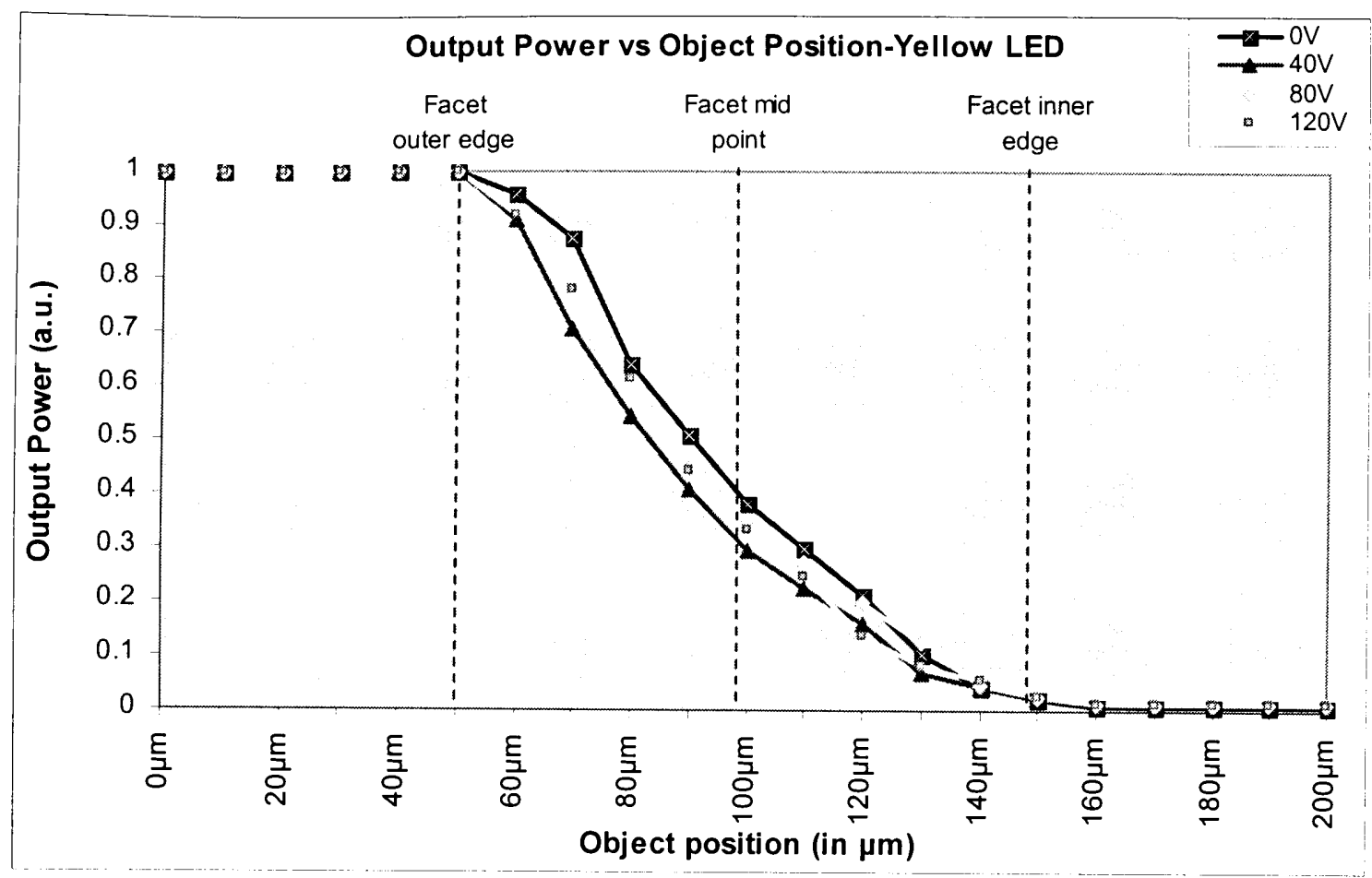

Figure 10: Variation in power coupled as a knife-edge is inserted in the gap. Curves are plotted for 4 different gap sizes corresponding to actuator voltages of $0 \mathrm{~V}, 40 \mathrm{~V}, 80 \mathrm{~V}$ and $120 \mathrm{~V}$.

From the results obtained, it can be concluded that the output does not vary significantly when the knife-edge is outside the gap. There is some change when it has not yet entered the gap, and most of the change occurs as it blocks the gap between waveguides. One important observation was that amount of drop in output power when the knife edge blocks the gap was more in the top half of the facet compared to the bottom half. Due to this phenomenon, it becomes important to image the facet of micro-gripper to check if the light is uniformly distributed across the entire facet. An optical simulation was performed also to check if there was more light present in the upper part of the facet compared to the lower part. A FDTD simulation was done[18] using RSOFT for SU8 refractive index $n=1.6$ and air cladding refractive index $n=1$, since the microgrippers are 
suspended in air. The simulation results show some losses due to the bending of waveguide and this leads to more light coming out of the top of the facet compared to the bottom part.
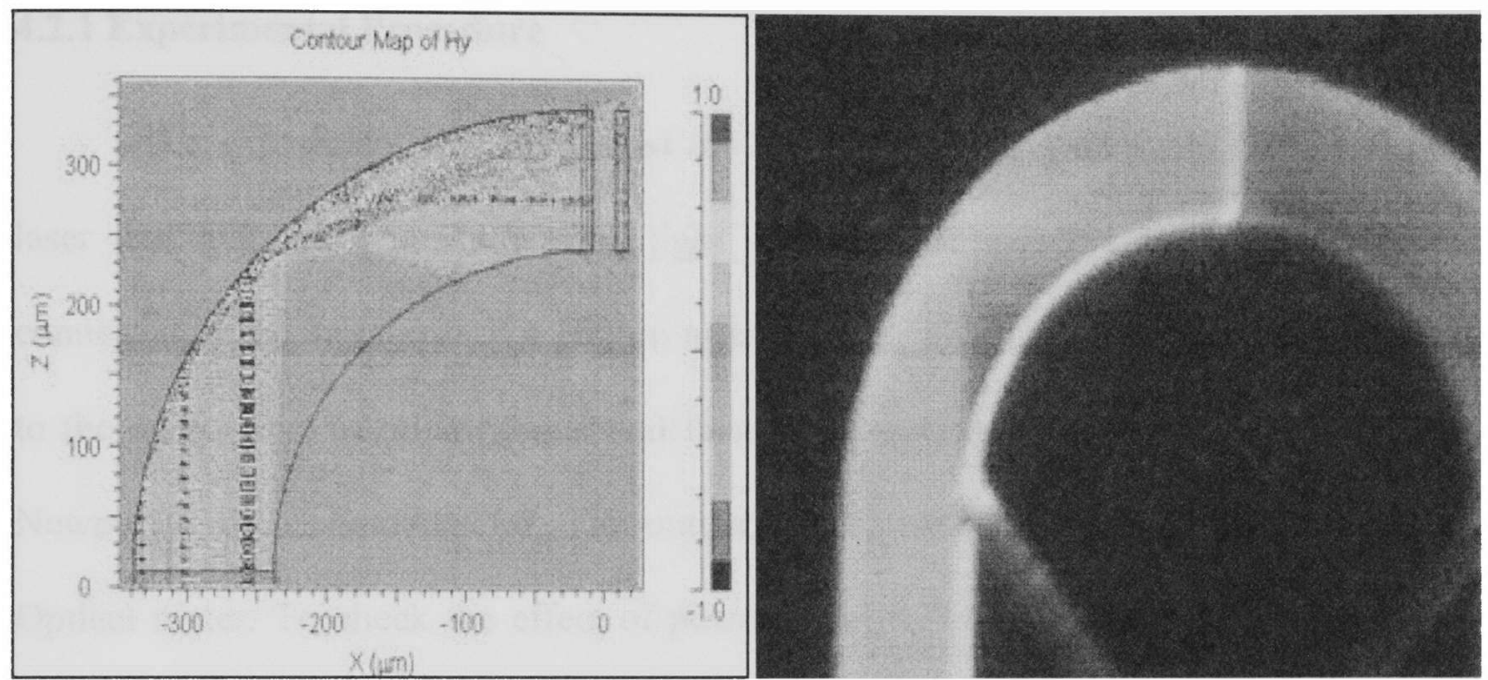

Figure 11: The figure shows the comparison between the simulation and the actual experiment for the bending losses in the waveguide[18].

The waveguide microgripper's ability to detect the insertion of an object between the gripper facets using light was demonstrated using this experiment. Knife-edge experiments show the operation of the gripper affects the power distribution across the facet and indicates the need for further research to engineer the optical fields at the facet.

\subsection{Power coupling between the facets}

To determine the effect of the distance between the microgripper facets on the total power coupled between them, an experiment was performed. From the experiments that were performed earlier, it was evident that the output power varies a lot for different light sources. The experiment was performed using 2 different light sources, a $532 \mathrm{~nm}$ green 
laser light and a 472nm Blue LED. Firstly the experiment was executed using green laser light.

\subsubsection{Experimental Procedure}

The experiment was performed for two different light sources, a $532 \mathrm{~nm}$ green laser and $472 \mathrm{~nm}$ blue LED. The light was coupled into the input fiber which is connected to the input arm of a $100 \mu \mathrm{m}$ microgripper. The light is guided from input arm to the output arm of microgripper and then to the output fiber which is connected to a Newport 818SL photodetector. The output can be obtained from the Newport 1835C Optical meter. To check the effect of power coupling between the facets for change in griping arms gap, a voltage source is connected to the piezo actuator. A function generator is used to trigger a triangular wave. The triangular wave leads to opening and closing of the gripper arms at a set frequency. The stub at the bottom of fishbone structure is subjected to pushing and pulling forces by the shaft that is connected to it by UV curable glue. The stub is pushed during the positive peak of triangular wave hence opening the gripper arms and pulled during the negative peak hence closing the gripper arms. A digital oscilloscope is used to monitor the variation in the output power when the gap between the gripping arms is varied from $0 \mathrm{~V}$ to $40 \mathrm{~V}$ which corresponds to varying the gap from $16 \mu \mathrm{m}$ (relaxed microgripper gap) to approximately $21 \mu \mathrm{m}(\sim 5 \mu \mathrm{m}$ increase) $[18]$. 


\subsubsection{Experimental Results}

Figure 12 shows the measured intensity of the transmitted optical power as the microgripper opens.

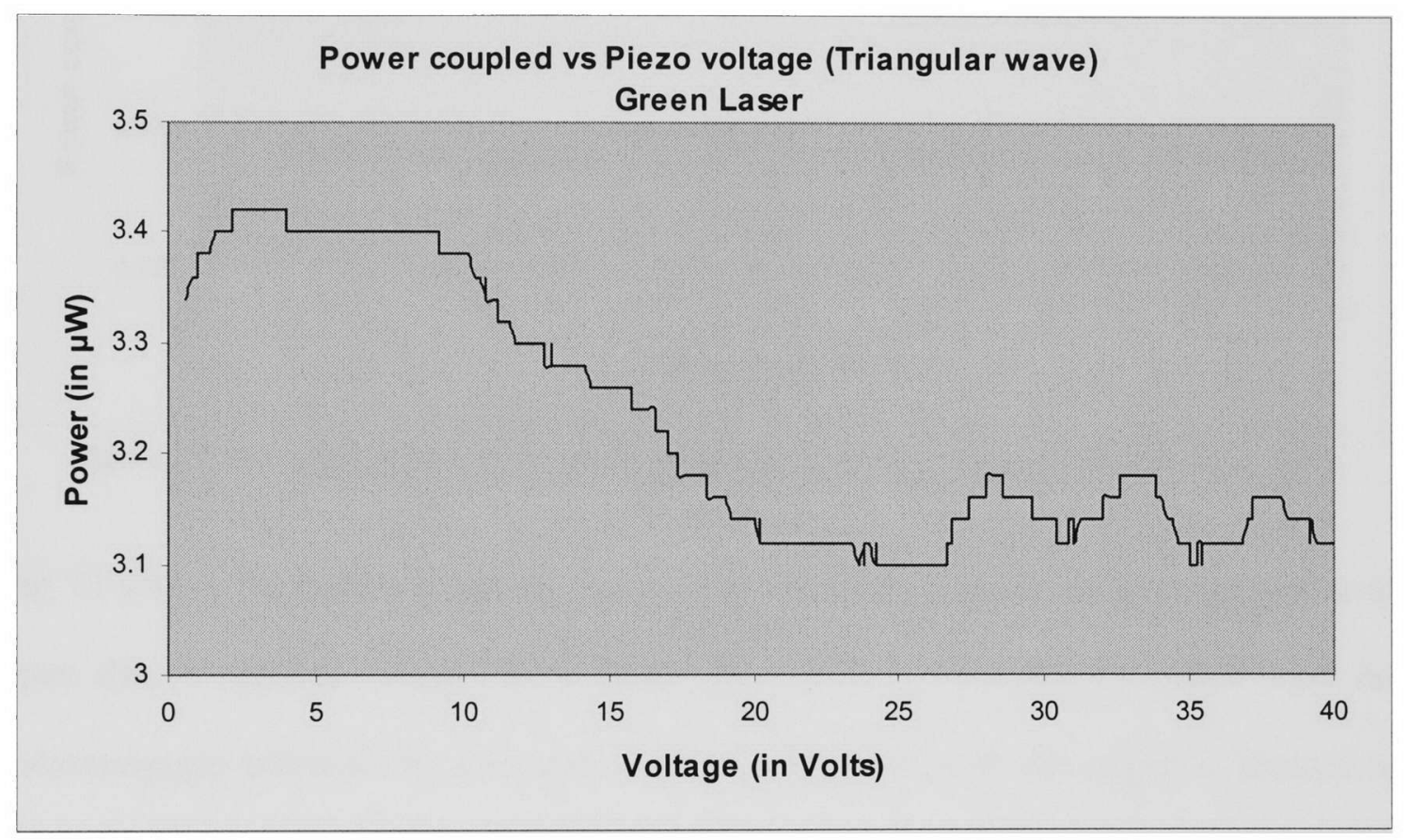

Figure 12: Transmitted power vs Voltage applied(leading to opening of arms) for green laser

From figure 12, it can be observed that as the microgripper facets separate, the power coupled between the facets is modulated, presenting a series of peaks. The factors leading to the observed transmission modulation are not certainly known, however, factors that could contribute to this phenomenon could be the non-perfectly vertical facets, coupled with the multimodality of the structure, which could produce constructive and destructive interference as the microgripper opens. The same experiment was executed using blue LED light source and the result was as seen in figure 13. 


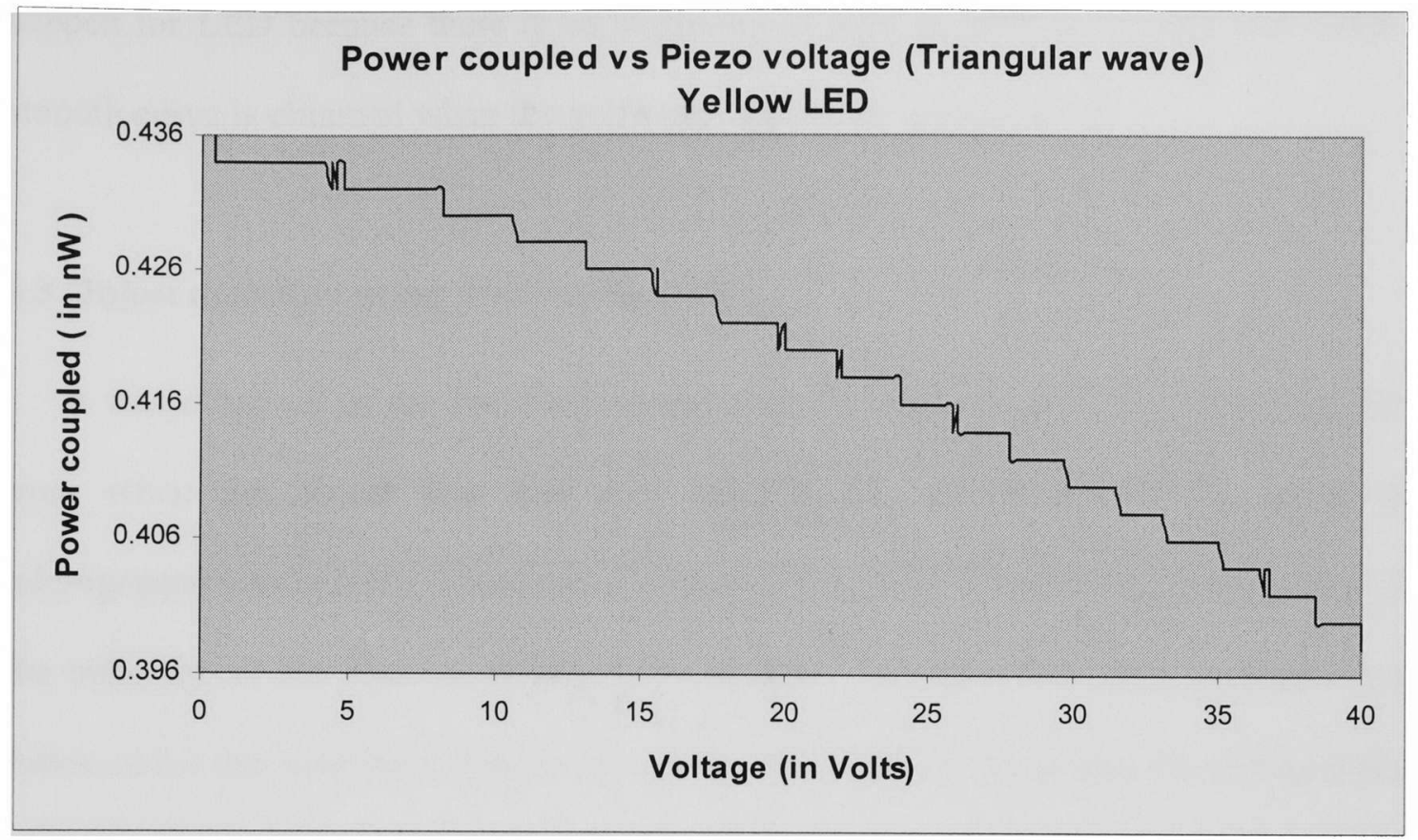

Figure 13: Transmitted power vs Voltage applied(leading to opening of arms) for blue LED

Fig 13 shows the measured intensity of power transmitted when the microgripper arms open due to applied voltage for a blue LED. A linear decrease in power with the increasing gap between the arms can be clearly seen. The step like output is because of the very low power output available for sensing. Hence the effect of noise is significant. Nevertheless the output power decreases gradually as expected. The amount of power drop at a given voltage is in agreement with the knife edge experiment explained previously. From Table 2 the drop in output power from $0 \mathrm{~V}$ to $40 \mathrm{~V}$ is calculated to be $7 \%$. From fig 13 the drop in output power from $0 \mathrm{~V}$ to $40 \mathrm{~V}$ is calculated to be $8 \%$. The same relation could not be established for the laser source because of the reflection of light from the knife edge. As seen in fig 8, there is a slight increase in power as the knife approaches the microgripper gap and this increase can be associated with the light reflecting from the knife and coupling into the output waveguide. And this doesn't 
happen for LED because there is no scattering of light in LED as in laser and hence a smooth curve is obtained when the knife approaches the gripper arms.

\subsection{Object detection using fiber to fiber setup}

It was observed in the knife experiment that the drop in light was more than $60 \%$ even when the object was half way through the microgripper facet. Since the microgripper waveguides are connected to the optical fiber, it becomes important to study the behavior of the fiber in transmission of light. Therefore the same procedure was followed but this time for a fiber to fiber setup which closely resembles the microgripper.

\subsubsection{Experimental Setup}

The setup consists of a light source(532nm Green Laser and 472nm Blue LED), two $125 \mu \mathrm{m}$ multimode optical fibers $(62.5 \mu \mathrm{m}$ core diameter $)$, Newport 3 DOF micropositioner, Newport 818SL photodetector, Newport 1835C Optical meter and a $20 \mu \mathrm{m}$ thick aluminum film. The two multimode fibers act as input and output waveguide similar to the input and output arms of a microgripper. The fibers were cleaved at one end. The cleaved tips of fiber face each other and the other end of input fiber goes to light source and output fiber goes to a photodetector. The light(Laser or LED) is passed through the input fiber and the output fiber is aligned using a 3 DOF micro-positioner such that maximum output is obtained. When this setup is ready, another micropositioner is used to hold the Aluminum film and the film is inserted in between the 2 aligned fiber tips to block the light. 


\subsubsection{Procedure}

Suppose the knife is moved from left to the right with respect to Fig 14. The initial position of the Aluminum film(knife) was $50 \mu \mathrm{m}$ away from the outer edge of the cladding. The knife is moved in the steps of $10 \mu \mathrm{m}$ towards the edge. The knife covers a total distance of $200 \mu \mathrm{m}$ covering $50 \mu \mathrm{m}$ outside the fiber, $125 \mu \mathrm{m}$ of fiber diameter and further $25 \mu \mathrm{m}$ inside the inner edge of cladding. The output power was measured at each step.

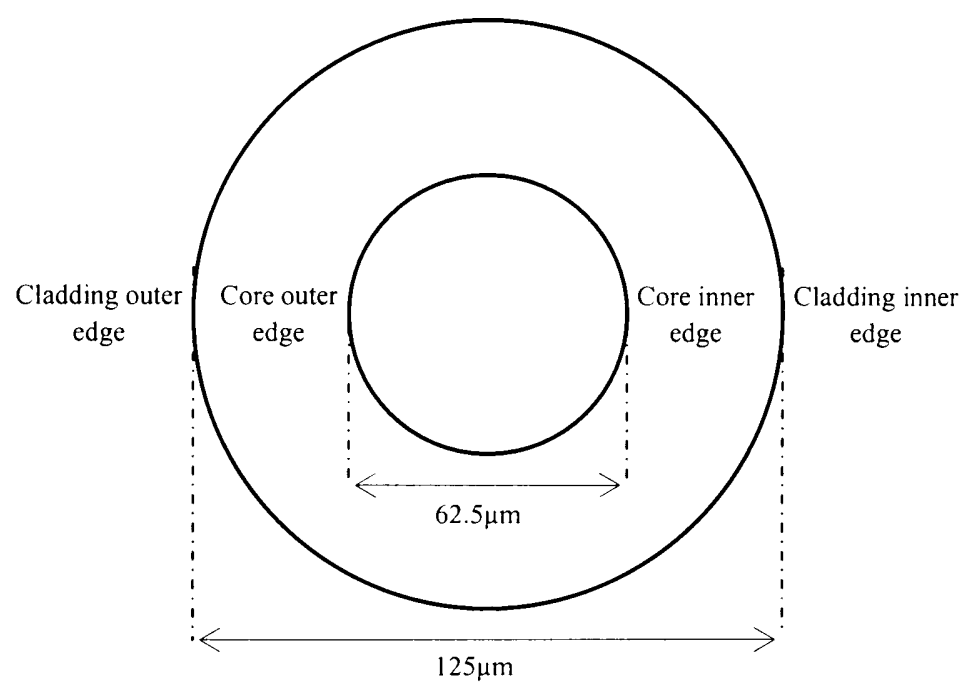

Figure 14: Layout of a tip of $125 \mu \mathrm{m}$ thick multimode fiber with $62.5 \mu \mathrm{m}$ core diameter.

\subsubsection{Experimental Results}

Fig 15 shows the measured output power as the object blocks the gap between the input and output fiber. 


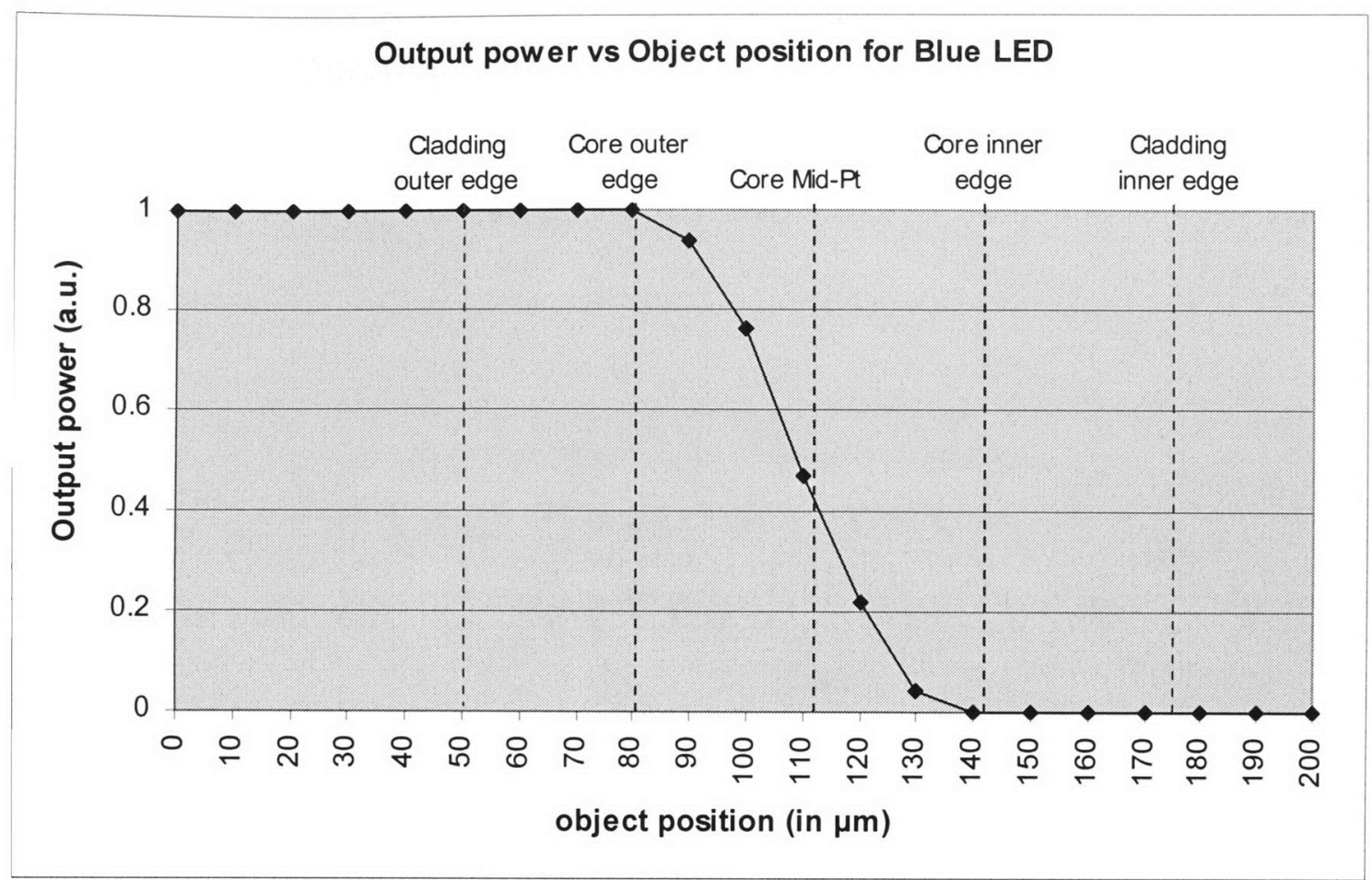

Figure 15: The graph shows the variation in power coupled as a knife-edge is inserted in the gap for a blue LED light source

The above curve clearly indicates no change in power until the knife reaches the outer edge of the fiber core. Core is where all the light is confined hence no change is observed from outer edge of cladding until the outer edge of core. When the knife is moved further in, the power begins to drop gradually with the increase in insertion distance. A power drop of approximately $50 \%$ is observed when the knife reaches mid-point of the core as expected. The output power drops to $0 \%$ when the knife reaches the inner edge of core. This implies that the light is almost uniformly distributed across the core surface. To verify the uniform distribution, the imaging of fiber tip for a blue LED was performed which will be discussed in next section. The object detection for fiber to fiber setup was demonstrated using green laser also. A very significant change was observed compared to the LED. The result was as seen in figure 16. 


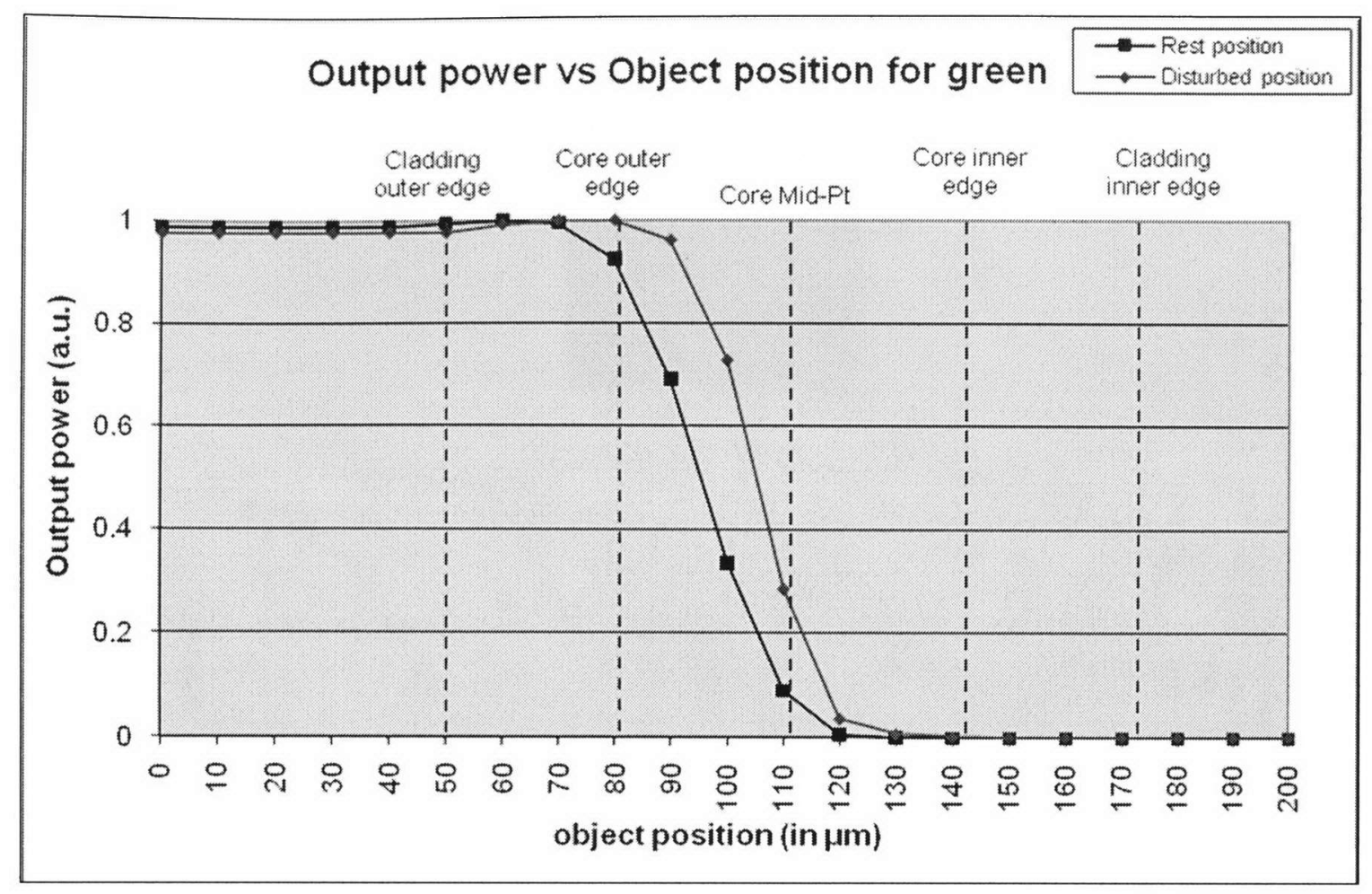

Figure 16: The graph shows the variation in power coupled as a knife-edge is inserted in the gap for a green laser light source

A very sharp power drop was observed from the region between the core outer edge and core mid-point. Consider the curve with no bending, it can be seen that almost $90 \%$ of the power is lost when the knife reaches the mid-point of the core. The reason for such huge drop is suspected to be diffraction which is being investigated as a part of an ongoing project in the group. This experiment was simulated using MATLAB to check if such a huge drop is practical. An image of fiber tip illuminated by green laser was taken converted to grayscale and it was blocked in steps. A row of pixels was covered in steps until the entire image was covered. This modeled the process of physically blocking a fiber tip using a knife. The blocking of pixels was done from 2 different directions to see if there is any change if the knife were to block from different direction. 


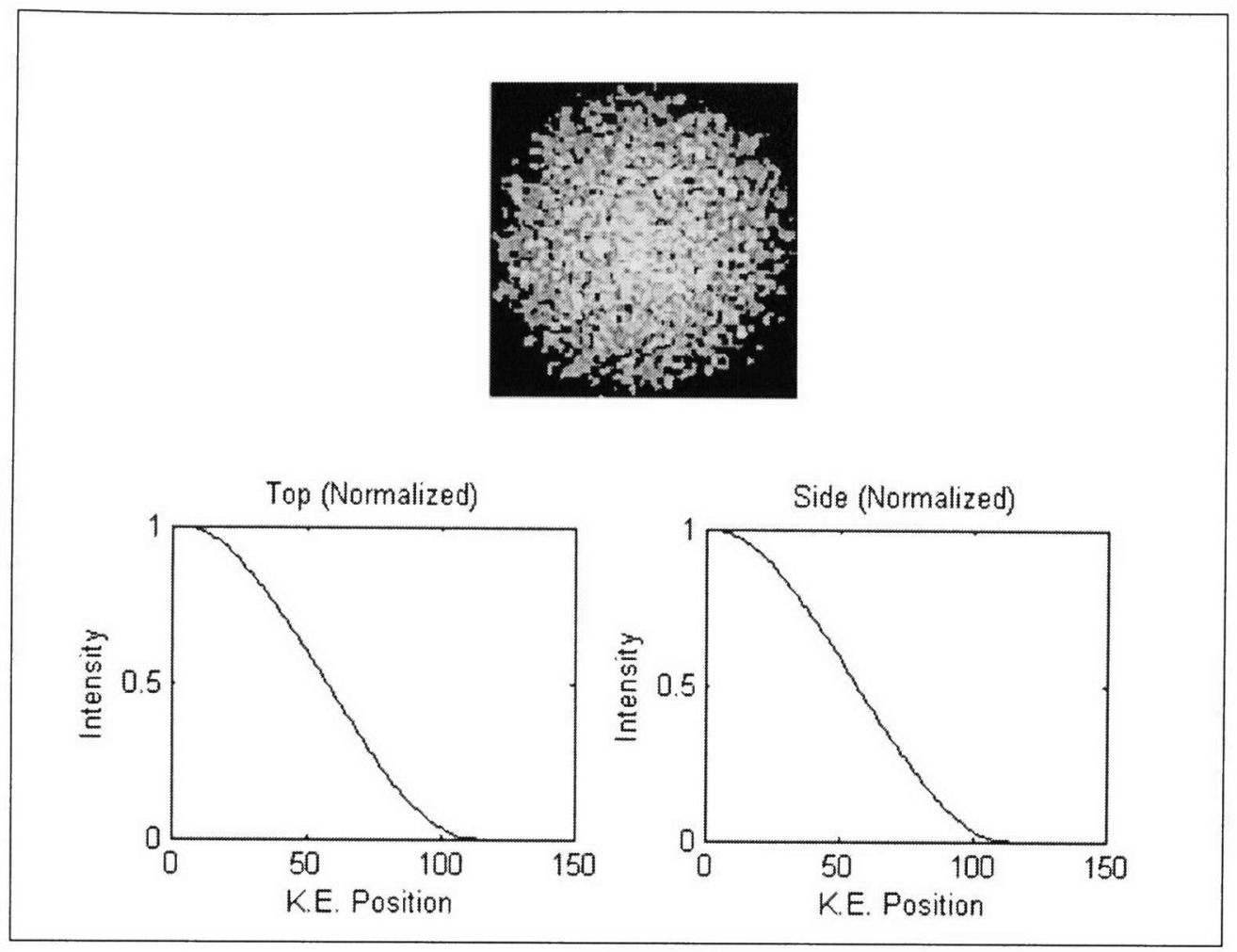

Figure 17: The grayscale image of fiber tip illuminated by green laser and the curves for output power vs knife position from two different directions.

A threshold of $45 \%$ was set; meaning any pixel which was less than 0.45 times the maximum intensity value was set to 0 . This way the background light was eliminated which could have been one of the reasons for huge drop in power seen in the actual experimental data. Figure 16 shows the grayscale image of the laser illuminated fiber tip which was blocked in steps by covering rows of pixels. The curves represent the output power coupled vs. the position of the knife. The results show $\sim 55 \%$ drop at the midpoint of core which is close to the ideal value expected. Also in the experimental data it was seen that the amount of power coupling changes when the fiber is disturbed. This will be further investigated by taking images of the fiber facet as explained in the following section. 


\subsection{Imaging}

The object detection ability of microgripper was discussed above and the results obtained led to some speculations like light is not uniform across the facet and also the modes are not distributed uniformly. But these speculations need to be justified with some results. That made it very important to perform the imaging of the microgripper facet. To image the microgripper, output side of an assembled microgripper was taken apart and the input side was retained. A CCD was setup in front of the input facet to grab the images of illuminated facet.

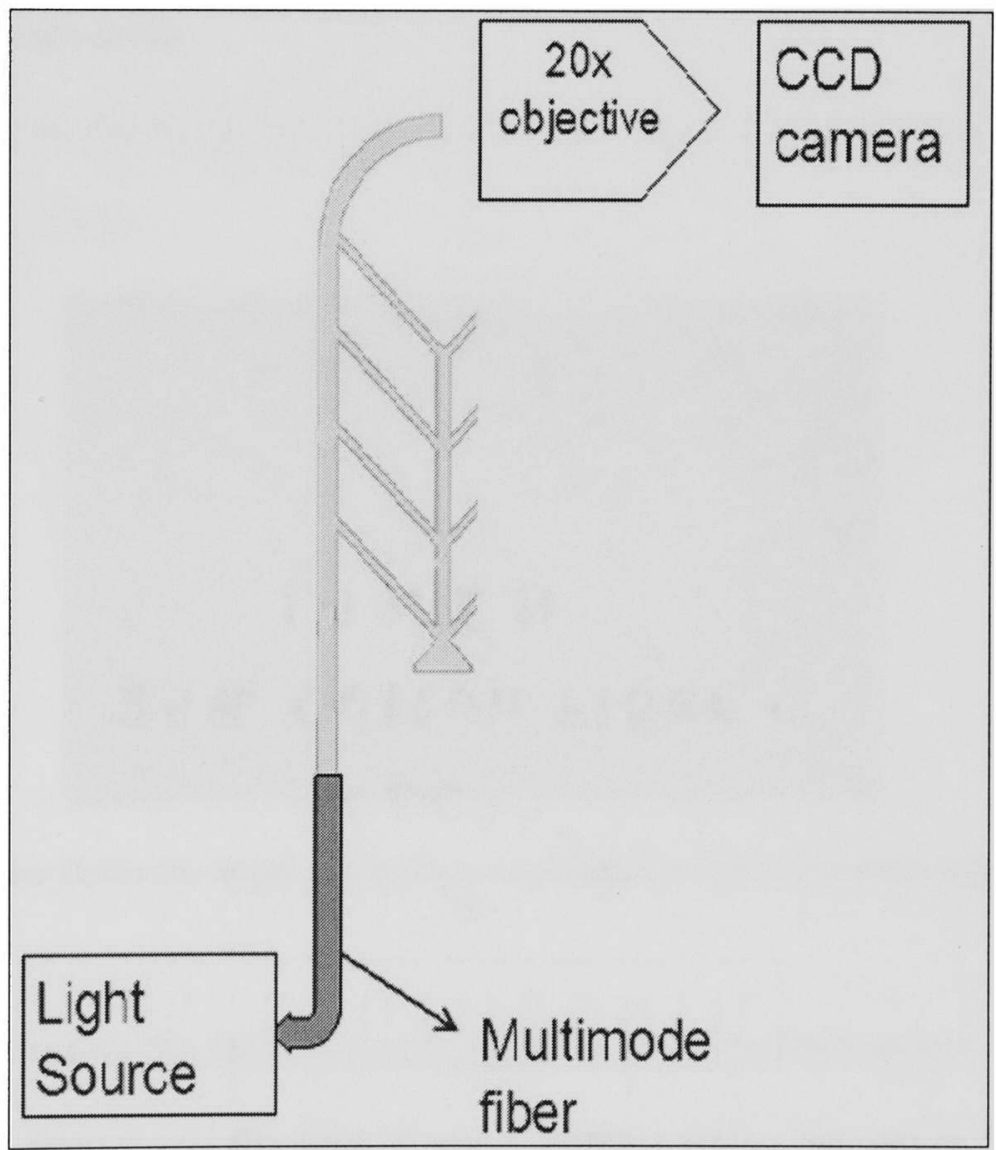

Figure 18: Schematic of imaging a microgripper facet by a CCD camera. 


\subsubsection{Experimental Procedure}

The first half of setup is same as the basic microgripper setup. Light is coupled through the multimode input fiber. For better coupling, the light is focused using a $20 \mathrm{x}$ objective. The light travels through the input waveguide and instead of coupling to the output waveguide, this light is focused on a CCD since the output part of the microgripper is taken apart. To better image the facet, a 20x objective was used and the CCD could grab magnified images of the microgripper facet.

\subsubsection{Experimental results}

Fig 19 shows the image of $50 \mu \mathrm{m}$ microgripper facet magnified by a $20 \mathrm{x}$ objective and focused onto a CCD

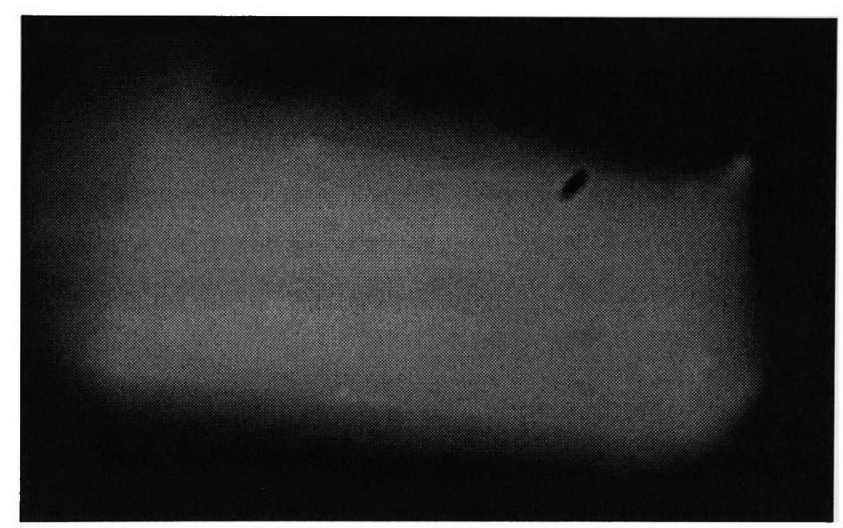

Figure 19: The figure shows the image of the $50 \mu \mathrm{m}$ microgripper facet with white light coming out of it.

The above image shows the facet of microgripper without the laser source. The image is for a white light source and the light is quite uniform across the entire facet. But the purpose of this experiment was to show the multiple modes coming out of the facet and also the non uniform distribution of the facet. The white light was replaced by a $532 \mathrm{~nm}$ 
green laser and a series of images was taken. To see the variations in the modes, the fiber was disturbed in various ways like pressing, rotating and twisting. The images clearly showed the shifting of modes as expected.

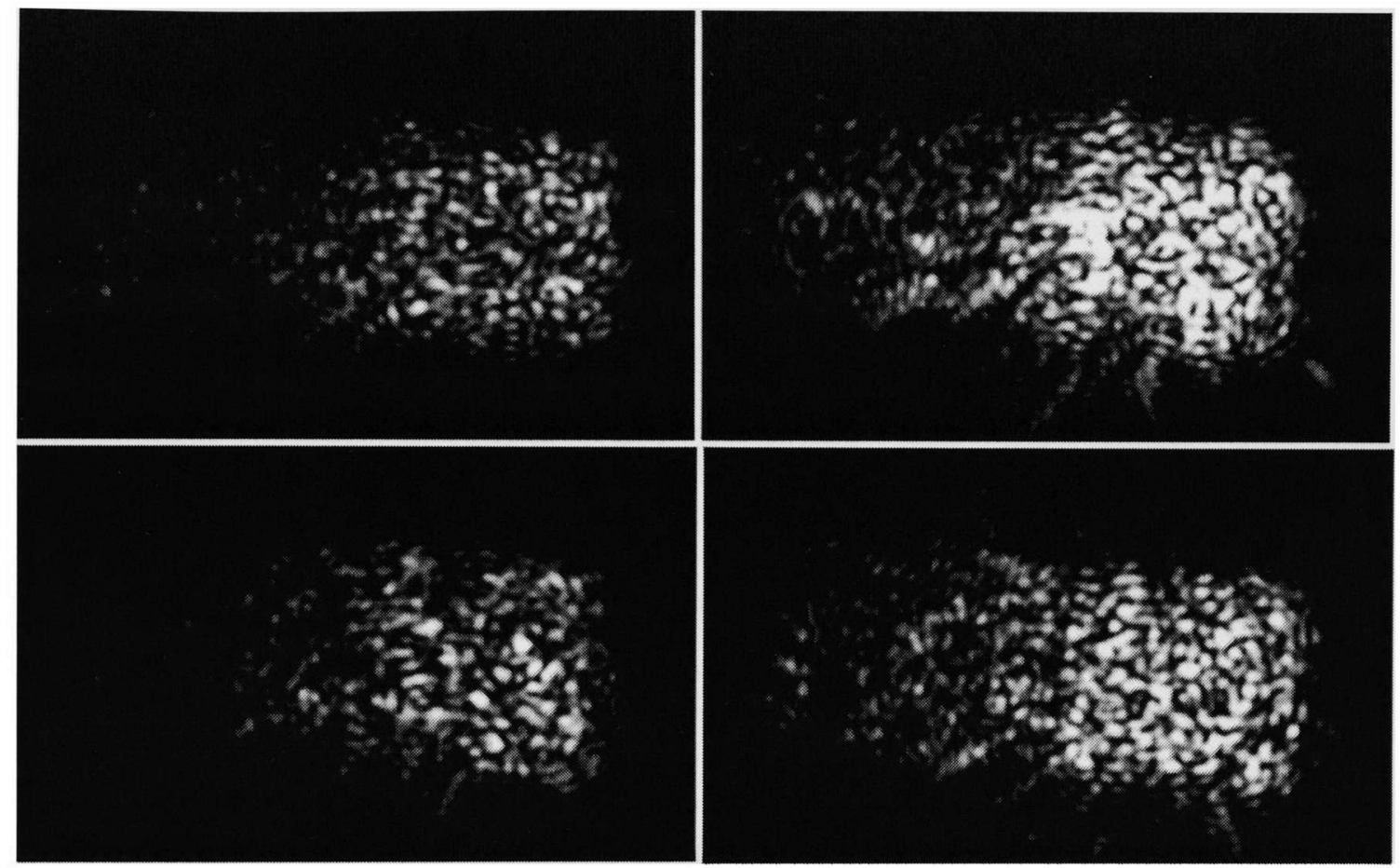

Figure 20: The series of images show the facet of microgripper captured by CCD for different positions of input fiber like rotation, pressing and twisting. The change in mode distribution can be clearly seen.

The images of the microgripper facet shown in figure 20 are for fiber under different conditions. The input fiber was messed up and the pictures were taken. The pictures imply that the modes are not distributed uniformly. To find out what leads to this uneven distribution of modes, the cleaved tip of a multimode fiber was imaged in the same way as the microgripper facet. The light was coupled to a multimode fiber exciting all the possible modes. When fiber modes interfere, they create a speckle pattern of constructive and destructive interference that moves across the detection area.[25] 


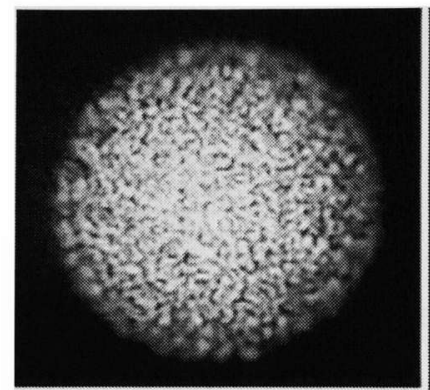

$\boldsymbol{a}$

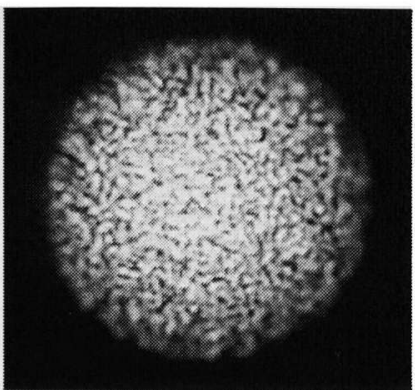

$b$

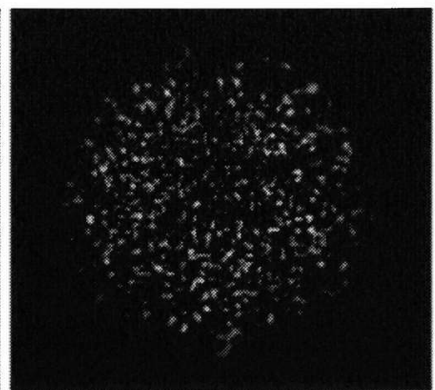

$c$

Figure 21: Image a shows the multimode fiber tip illuminated by green laser. Image $b$ shows the same fiber but with perturbation. Image $c$ shows the difference between images $a$ and $b$.

Fig 21a shows the image of tip of a multimode fiber in rest position illuminated using a $532 \mathrm{~nm}$ green laser. Fig $21 \mathrm{~b}$ shows the image of tip when the fiber is messed up. Fig $21 \mathrm{c}$ shows the difference between fig $21 \mathrm{a}$ and fig $21 \mathrm{~b}$ and it clearly indicates that mode distribution changes significantly even for a slight perturbation in fiber. The same experiment was performed for blue LED and the results were very different from the laser as expected. The illumination across the fiber tip is almost uniform.

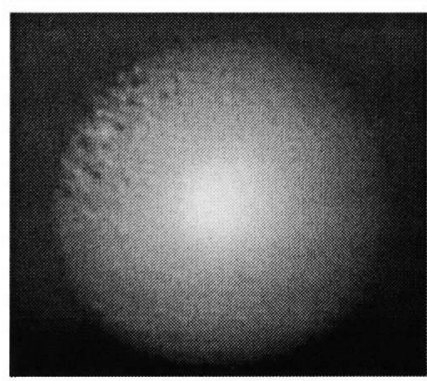

$a$

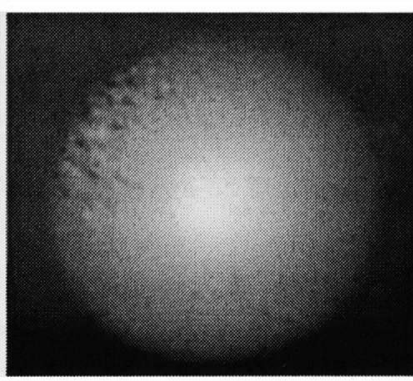

$b$

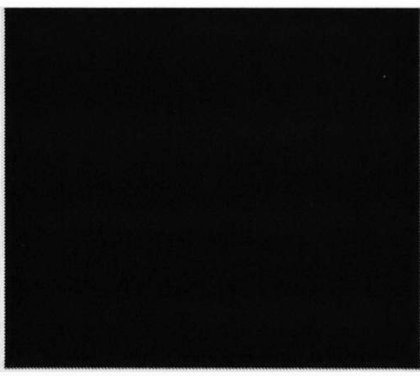

$c$

Figure 22: Image a shows the multimode fiber tip illuminated by blue LED. Image b shows the same fiber but with perturbation. Image $c$ shows the difference between images a and $b$.

Fig $22 \mathrm{a}$ shows the image of fiber tip illuminated using blue LED. Fig $22 \mathrm{~b}$ shows the image of the same fiber tip but for a perturbed fiber. Fig $22 \mathrm{c}$ shows the difference between images $a$ and $b$. The subtraction of image $a$ and $b$ is zero which shows that there is no change in modes in case of LED and also the illumination is uniform across the tip. 


\section{FLUORESCENCE DETECTION}

\subsection{Introduction}

Fluorescence is a luminescence that is mostly found as an optical phenomenon in cold bodies, in which the molecular absorption of a photon triggers the emission of a photon with a longer (less energetic) wavelength. Fluorescence occurs when a molecule, atom or nanostructure relaxes to its ground state after being electrically excited.

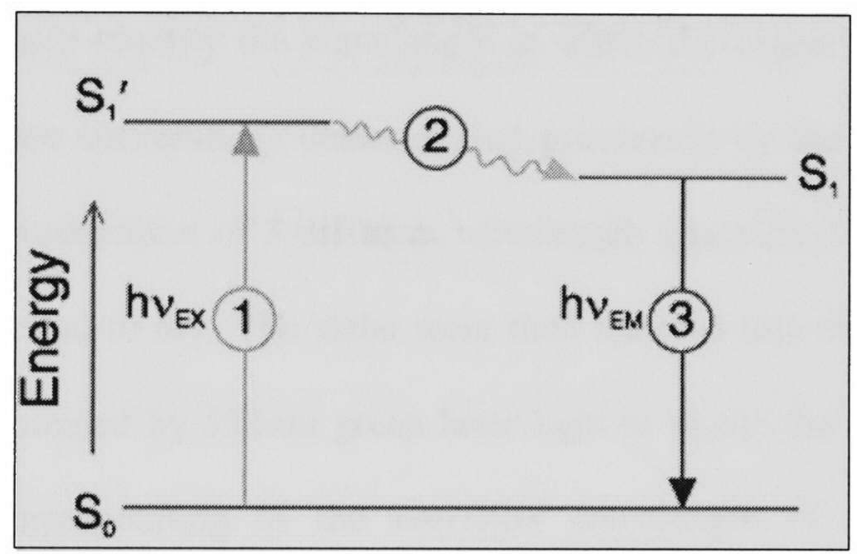

Figure 23: Mechanism of fluorescence emission.

The above figure explains the process of fluorescence emission. The electrons present in the ground state $S_{0}$ are stimulated and they jump to the excited state $S_{1}$ ' by absorbing the energy. The electrons stay in the excited state for a very short time and they move to an intermediate energy state $S_{1}$ by releasing some amount of energy. Now when the electrons from this intermediate state fall back to the ground state, they lose all the energy that they gained and this energy difference is emitted in the form of fluorescence. The experiments performed in this work make use of quantum dots which were excited using 
$532 \mathrm{~nm}$ green laser light and UV light and the objective was to detect the fluorescence emitted by the quantum dots.

\subsection{Quantum dot tagging}

As mentioned before, the microgripper has the integrated optical feedback. It can collect the light emitted from the microobject under study and this light can help study various properties of the structure under study. One of the properties is to identify the fluorescence of the object. The output of microgripper when connected to the spectrometer will help identify the wavelength at which the object fluoresces. Quantum dot tagging has been successfully demonstrated previously by the Martinez et al. [18] where a known concentration of 3 different wavelength quantum dots were poured onto SU8 slabs and allowed to dry. The slabs were then inserted into the microgripper arms gap and were illuminated by $532 \mathrm{~nm}$ green laser light to excite the quantum dots. Three different peaks corresponding to the emission wavelength of quantum dots were distinctly identified. Figure 24 shows the 3 different peaks obtained corresponding to the wavelength of the quantum dots used. The graph is normalized to the maximum intensity. The integration time used was $500 \mathrm{~ms}$. It is seen that all the peaks have more or less same intensity and bandwidth. The curve for $555 \mathrm{~nm}$ quantum dot looks a little asymmetric because of the $550 \mathrm{~nm}$ high pass filter used for the experiment. This quantum dot tagging experiment proved that the microgripper is capable of detecting the fluorescence. What needs to be studied is the minimum amount of quantum dots that the microgripper can sense. 


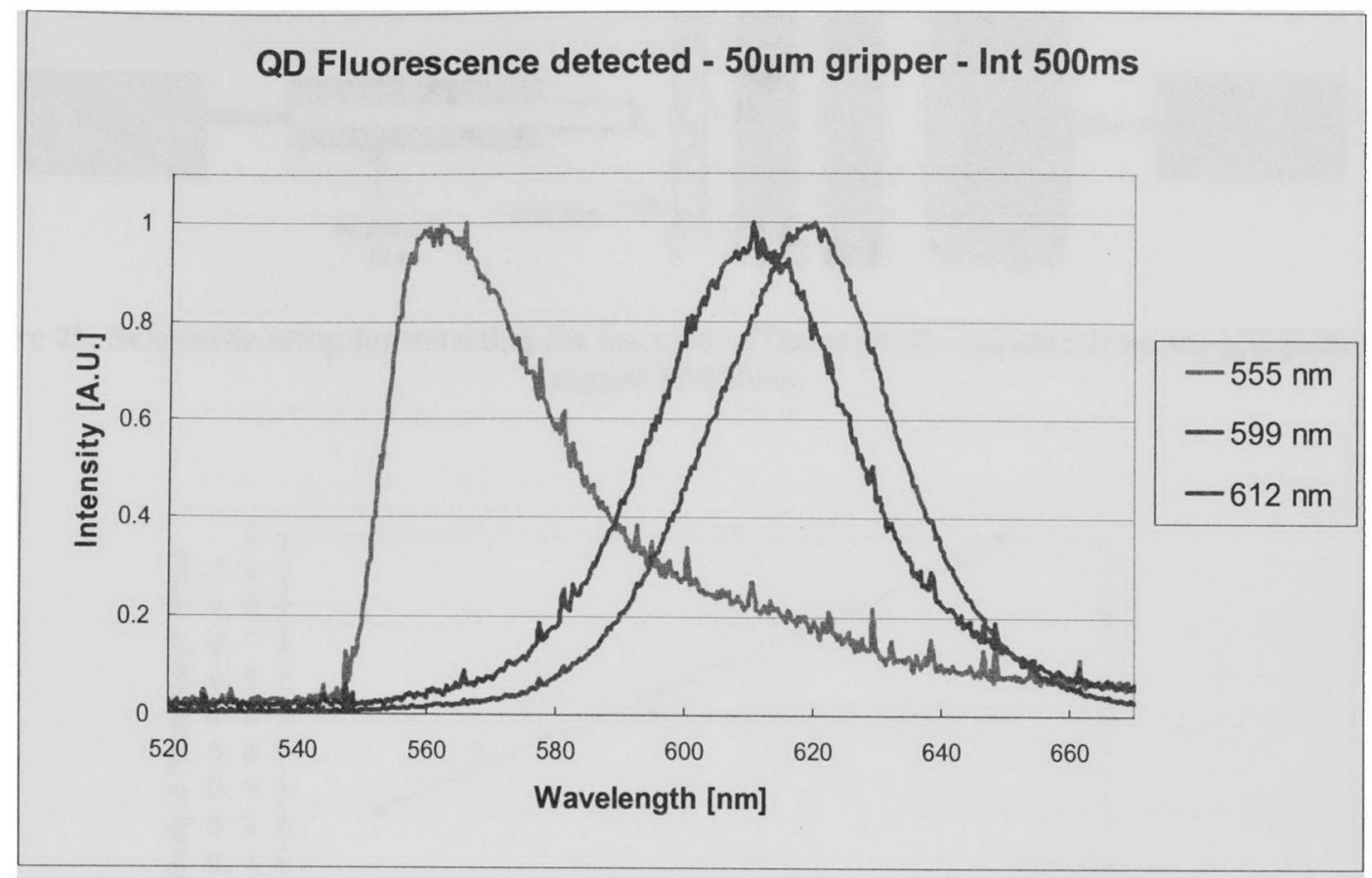

Figure 24: Fluorescence detected from SU-8 microparts tagged with QDs with emission at 555, 599, and $612 \mathrm{~nm}$ with an integration time of $500 \mathrm{~ms}$ [18].

To determine that, 4 SU8 films were spin coated with $600 \mathrm{~nm}$ quantum dots in different concentrations. This was done by doping the known amount of SU8 polymer with a known volume of quantum dots. The mixed solution was subjected to pre-baking, spinning, post baking and photolithography. The films released were $25 \mu \mathrm{m}$ thick and the concentrations in quantum dots per gram of SU8 were $4.36 \times 10^{14}, 2.26 \times 10^{14}, 1.16 \times$ $10^{14}$ and $5.73 \times 10^{13}$ respectively. An experiment was performed to check if the relation between fluorescence intensity and quantum dot concentration is linear. The SU8 films were illuminated by multimode fiber and the fluorescence emitted was collected by a photo-detector which was connected to an optical meter for measuring the intensity. A $532 \mathrm{~nm}$ notch filter and $600 \mathrm{~nm}$ band pass filters were used to eliminate laser and other unwanted light. 

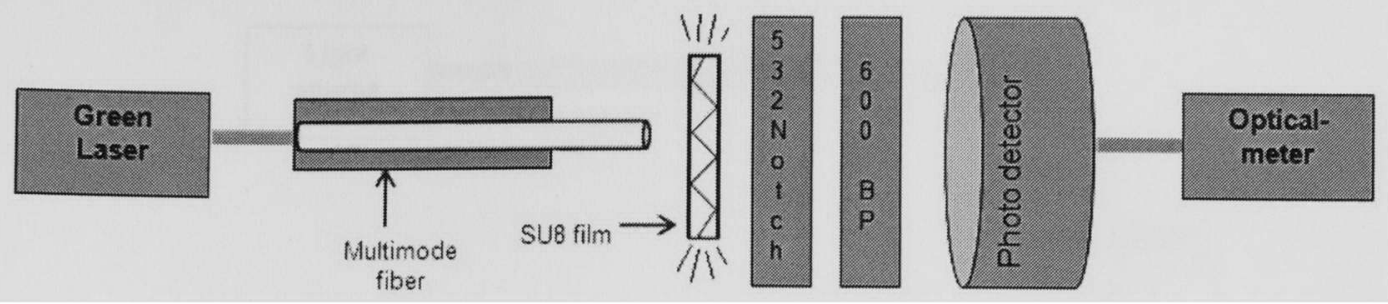

Figure 25: Schematic setup for detecting the intensity of fluorescence emission from the quantum dot tagged SU8 films.

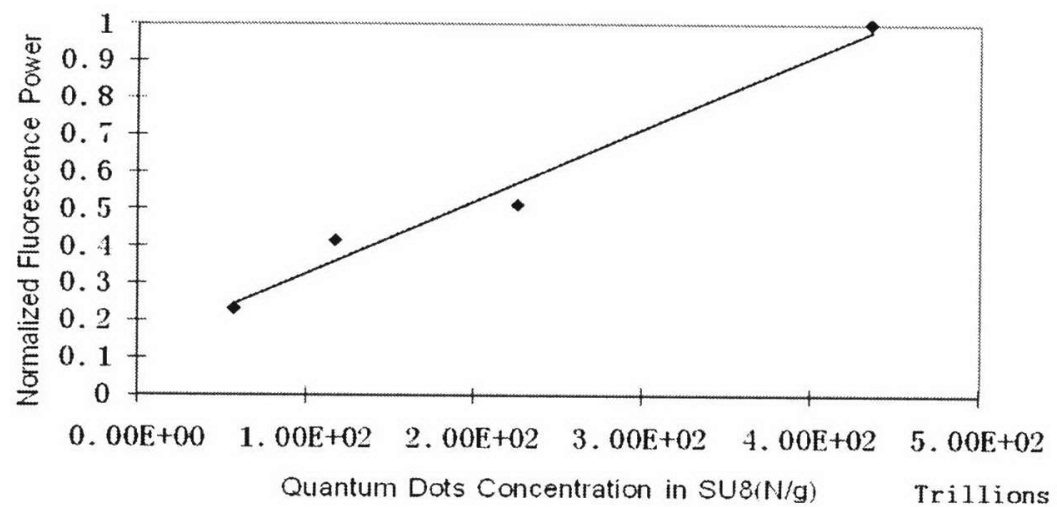

Figure 26: Curve representing the relation between Fluorescence intensity and quantum dot concentration.

The curve obtained in figure 26 shows that fluorescent intensity is linearly proportional to the quantum dot concentration the SU8 films. To obtain the fluorescence spectrum for these films, the experiment was setup as explained in section 5.2.

\subsection{Experimental setup}

The setup consists of a light source, an assembled microgripper, 600nm Quantum dots (evidots, Evident Technologies) tagged SU8 film, Newport XYZ micro-positioner, Ocean Optics USB 2000 spectrometer and a computer. 


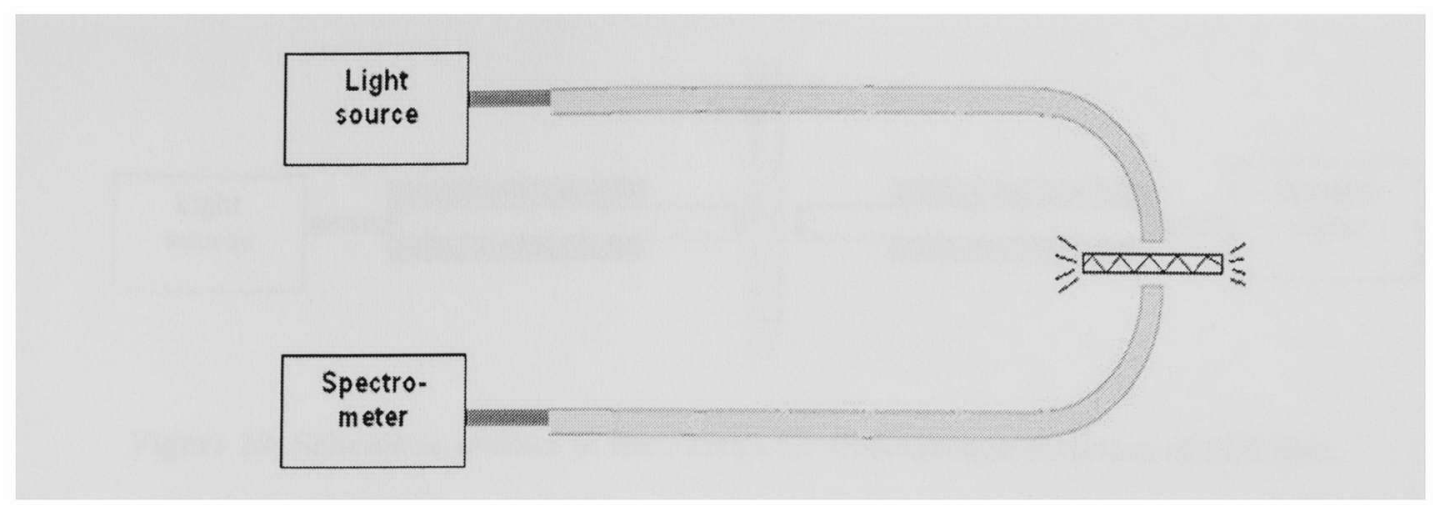

Figure 27: Schematic setup of fluorescence detection from a quantum dot tagged SU 8 film.

Depending on the light source, the input setup varies. The light is coupled into the input fiber which guides the light into the input arm of the microgripper. Quantum dot tagged SU8 films were inserted in the gap between the input and output arm of the gripper. The light coming through the input arm would then illuminate the inserted SU8 films. The output is connected to a spectrometer.

\subsubsection{Experimental Results}

The experiment was performed for 2 different light sources, a $532 \mathrm{~nm}$ green laser and an Ultraviolet lamp. All 4 SU8 films were tested in turns for both the light sources, but the fluorescence could not be detected by the microgripper. It was surprising to not have any fluorescence detected because it could be actually seen with the naked eye. Assuming that gripper was not capable of detecting this fluorescence, the same experiment was repeated using a fiber to fiber setup which very closely resembles the microgripper operation but with fewer losses. Two multi-mode fibers were perfectly aligned using $\mathrm{XYZ}$ stages and the distance between the fiber tips was set to be $30 \mu \mathrm{m}$. 


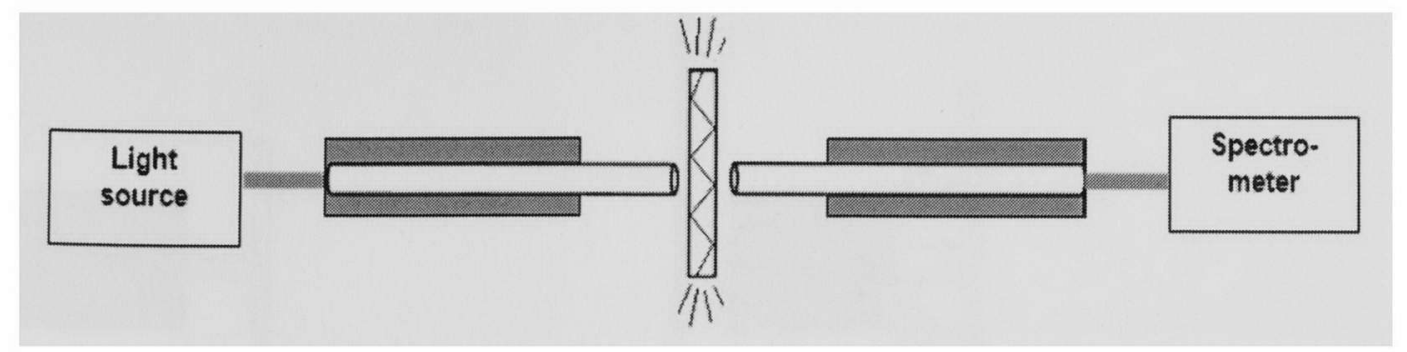

Figure 28: Schematic of fiber to fiber setup for fluorescence detection of SU8 film.

Similar to the microgripper experiment, the fluorescence was not detected for this setup either. But the fact is the light was seen guiding through the sidewalls rather than coming out of the surface. Hence the fluorescence was gone un-detected even though it was present.

\subsection{Modified setup}

To make sure that the fluorescence seen with the eye is because of quantum dots, the films were illuminated using both the light sources in turns and this time the output fiber was placed in a position to collect the light coming out of the edges of the SU8 films. The fluorescence detection from the edges could not be done using microgripper because of the limitation on gripper arms opening. Hence the setup was modified in such a way that the fluorescence could be collected from the edges of the films. Figure 29 shows the schematic of the modified setup to detect the fluorescence. The films were illuminated by the light source and the spectrometer probe was placed in position to collect the light from the edges. 

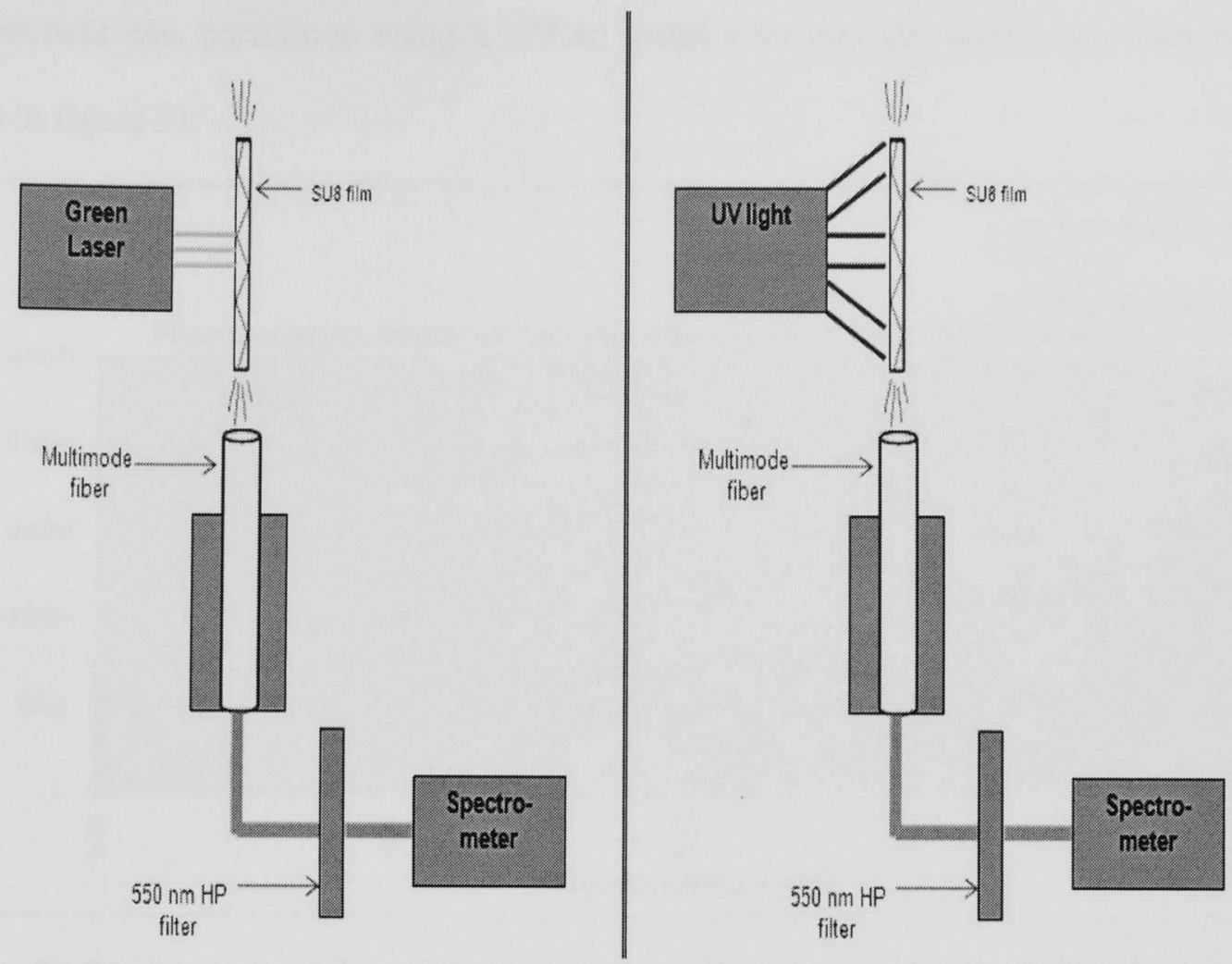

Figure 29: Modified setup to detect the fluorescence from the edges of the SU8 films. Setup on the left is for green laser light source and adjacent setup is for UV light source.

The major difference between the above 2 setups to be noted is the difference in the area of films illuminated by laser source and UV source. The consequence of this is discussed later in the chapter.

\subsubsection{Experimental results}

As expected, the fluorescence was detected and the spectrum was as seen in figure 30. Figure 30 shows the spectrum for 4 different concentrations of $600 \mathrm{~nm}$ quantum dot doped SU8 films. It can be seen that the peak varies corresponding to the concentration of quantum dots. A $550 \mathrm{~nm}$ high pass filter was used to eliminate the UV light. The same 
experiment was performed using a $532 \mathrm{~nm}$ green laser and the output spectrum was as seen in figure 31.

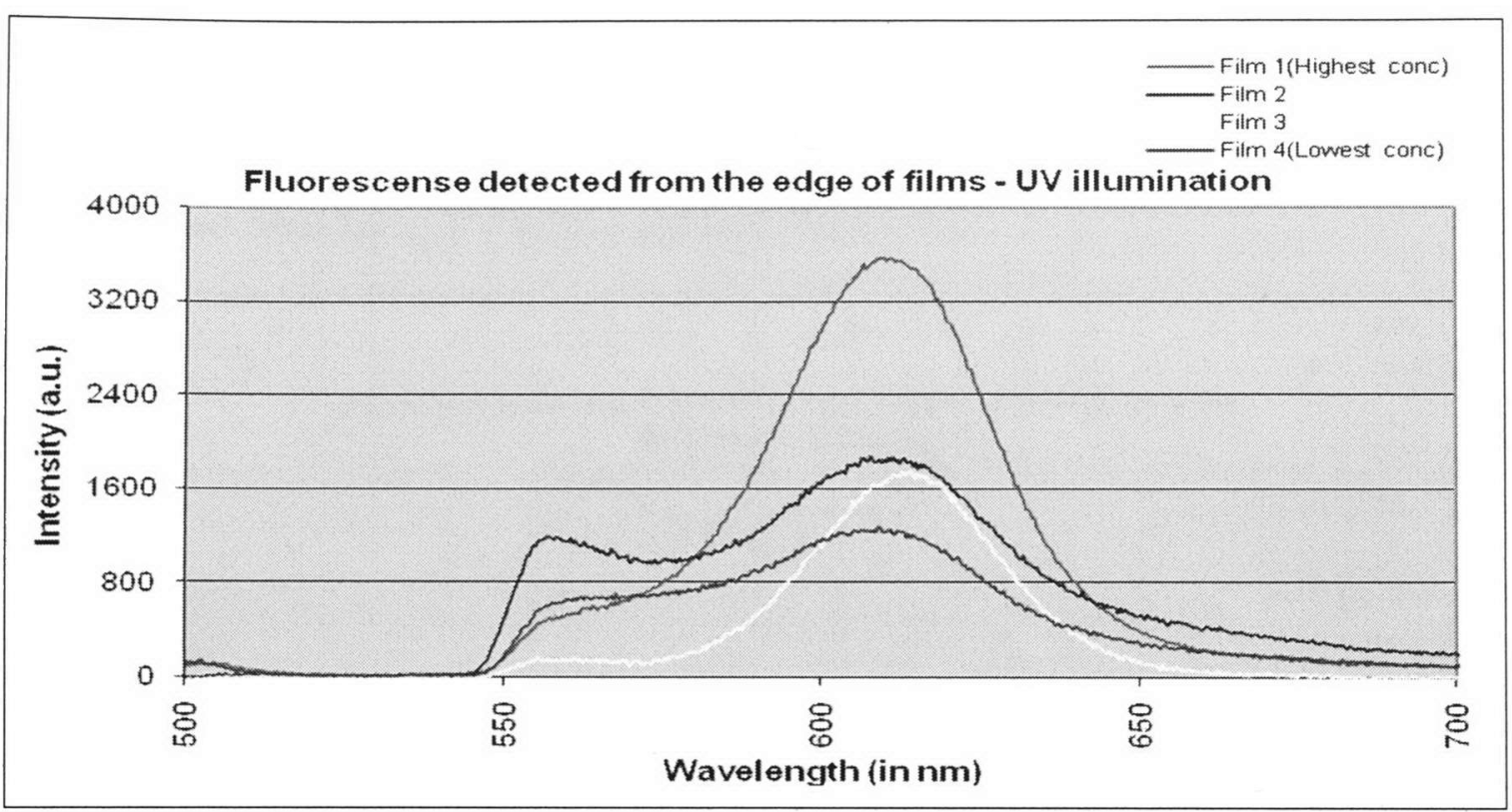

Figure 30: The graph shows the spectrum obtained for fluorescence emitted by $600 \mathrm{~nm}$ Quantum dot doped SU8 films of 4 different concentrations illuminated by UV light source.

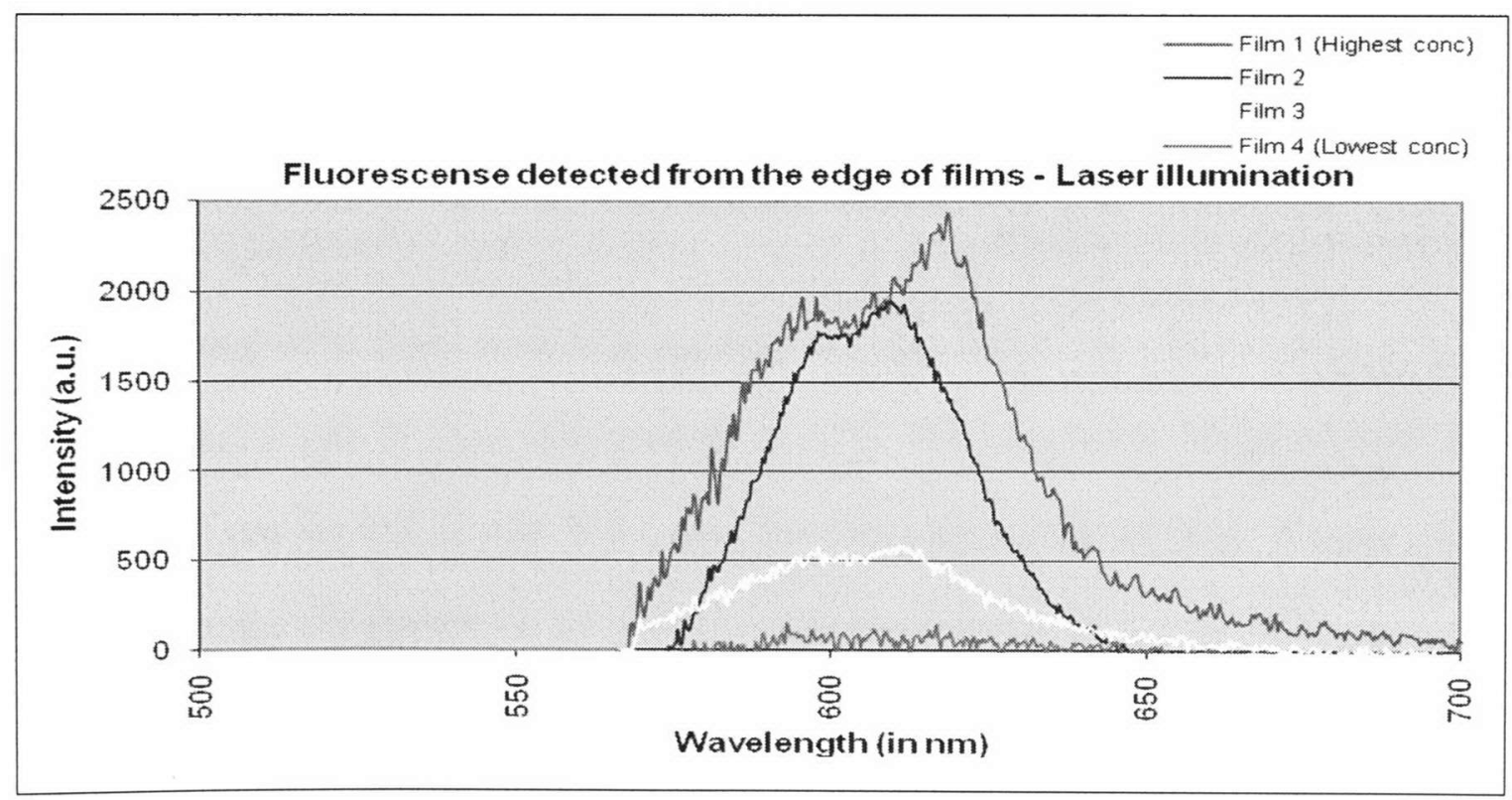

Figure 31: The graph shows the spectrum obtained for fluorescence emitted by $600 \mathrm{~nm}$ Quantum dot doped SU8 films of 4 different concentrations illuminated by green laser light source. 
The spectrum obtained for the laser and UV differs in 2 key aspects. First, the UV illumination leads to a peak at $560 \mathrm{~nm}$, which has been attributed to the fluorescence of the SU8 material [18]. Secondly, the laser illumination presents a dip in the quantum dot peak. Figure 32 shows the normalized curve for the fluorescence measure for highest concentration films using UV source and laser light source.

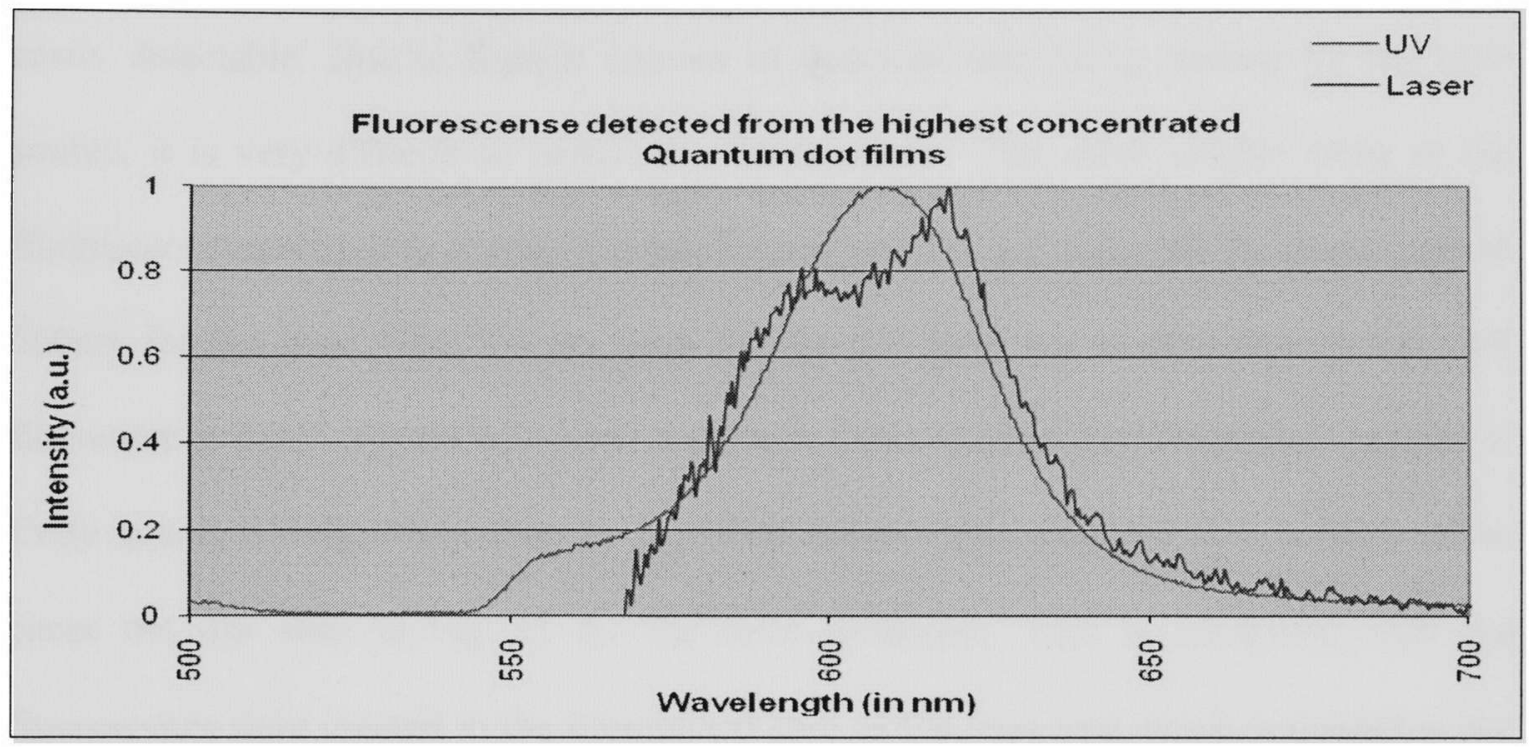

Figure 32: The figure shows the normalized curves for fluorescence intensity measured for highest concentration $600 \mathrm{~nm}$ Quantum dot tagged films for UV and laser source.

The width of both curves and main peak position is almost same which is expected. But different integration times were used because the intensity of fluorescence available for sensing was lot less in laser compared to the UV. The integration for green laser was $1000 \mathrm{msec}$ and for UV it was $100 \mathrm{msec}$. Therefore the curve for laser is noisy when compared to the UV curve. 


\subsubsection{Discusssion of Fluorescence}

A key difference in the experiment is that the laser illumination covers a small region of the sample due to sharp focus, far from the edge whereas the UV light illuminates the entire sample. Fewer quantum dots are excited by the laser compared to the UV illumination. Therefore the fluorescence intensity is higher for the UV source and it is easily detectable. Due to limited number of quantum dots being excited by the laser source, it is very difficult to collect the fluorescence. The other notable thing in the fluorescence spectrum is a peak around $550 \mathrm{~nm}$ for UV and a dip in the peak close to $600 \mathrm{~nm}$ for the laser. The $550 \mathrm{~nm}$ peak for the UV spectrum is attributed to the SU8 fluorescence [18]. Figure 33 shows the photoluminescence and absorption spectra of CdSe quantum dots. Absorption by non-illuminated CdSe quantum dots is believed to cause the dip seen in Fig 32 for the laser excitation. This is consistent with the fluorescence light created in the illuminated spot of the laser and which is guided in the waveguide through the unexposed regions, suffering wavelength dependent absorption.

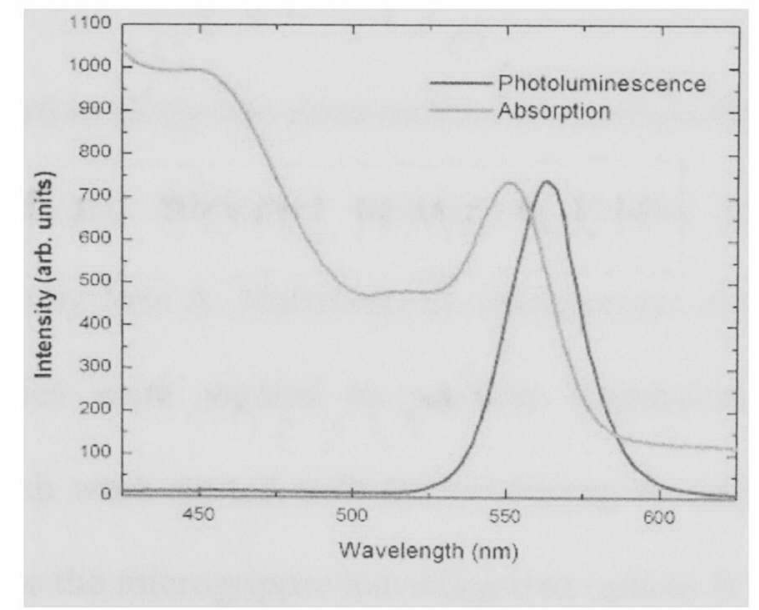

Figure 33: Absorption and photoluminescence spectrum of a CdSe nanocrystal[26], 
[27]. The result obtained for the laser illumination closely resembles the standard absorption curve in fig 33(b). It is believed that the quantum dots which are not illuminated by the laser light (dark quantum dots) absorb the fluorescence emitted by the illuminated quantum dots. Therefore not all the light comes out of the edges of the film and hence we see a dip in the curve. This phenomenon does not occur for UV illumination because all the quantum dots in the sample are illuminated hence there are no dark quantum dots present. Therefore all the fluorescence emitted by the quantum dots present in the sample is collected by the spectrometer which also justifies the intensity being more for UV than for laser.

\section{SUMMARY AND FUTURE WORK}

\subsection{Summary and Conclusions}

In this work, further study was done on the novel Micro-Opto-Electro-Mechanical which was developed and fabricated entirely at Florida International University nanofabrication facility by Jose A. Martinez[18]. Background of optical waveguides and its important properties were studied to provide foundation for the experiments performed. The research work started with demonstrating the object detection ability of the mocrogripper. Since the microgripper has integrated optical feedback, the presence of a microobject can be sensed by monitoring the output power. A drop in output power would mean that some object is blocking the gap. Knife edge experiment was performed 
to physically simulate this experiment. In this experiment, the gap between the microgripper arms was blocked using a $20 \mu \mathrm{m}$ thick aluminum slab(knife edge). The knife edge was moved in steps of $10 \mu \mathrm{m}$ and power output was measured at each step. Output power dropped as the knife edge moved inside after each step. Interestingly, more power drop was observed for the top part of the gripper compared to the bottom part. A power drop of over $60 \%$ was observed at travel distance of half the facet length. Hence it was suspected that the power distribution across the facet is not uniform. To verify this, the facet of a $50 \mu \mathrm{m}$ microgripper was imaged. This was done by breaking the output side of an assembled microgripper and retaining the input side. A CCD was setup in place of the output waveguide so that the images of the illuminated facet could be taken. As suspected, it was seen that the illumination was not uniform across the microgripper facet. More light was seen coming out of the top part of facet. This was because of the bending in the waveguide arm which led to the modes bouncing off the bend. This phenomenon was previously demonstrated by FTDT simulations using RSOFT[18]. Since the multimode fibers are used in connecting the microgripper waveguides, it was also interesting to study the imaging of a fiber facet. The results showed that mode distribution is very non-uniform for the green laser source. Also it was seen that the mode distribution changed a lot even for a slight perturbation in the fiber. The same procedure was followed to image the fiber facet for a blue LED source. The image for blue LED was very uniform across the facet and also no change was seen when the fiber was subjected to perturbations. Next the fluorescence detection ability of microgripper was studied. This unique ability of microgripper was previously demonstrated [18] by doping SU8 films with 3 different wavelengths and successfully detecting the signals 
corresponding to the quantum dot emission wavelengths. In this work, an attempt was made to find out the minimum amount of quantum dots that can be sensed by the microgripper. This was done by spin coating SU8 films with different concentrations of $600 \mathrm{~nm}$ quantum dots and the films were inserted in between the microgripper gap to see if the fluorescence can be detected. None of the films yielded any output but the fluorescence was actually seen with naked eye and it was evident that the light was guiding along the sidewalls of the film rather than coming out of the surface. The was proved by detecting the fluorescence signal from the side walls of the films using a spectrometer, but could not be done in the microgripper gap of the limitation in widening of gripper arms. To summarize, the object detection and fluorescence detection ability of microgripper were studied. Also the power coupling and factors affecting the power distribution across the facet were studied.

\subsection{Future work}

The microgripper discussed in this work is one of its kind with the integrated optical feedback and its other unique capabilities. But there are few issues that need to be addressed in order to make this device more sophisticated and useful. The two major hurdles that need to overcome are packaging and actuation mechanism. With the current procedure, it takes few hours to assemble a microgripper and the final setup is very bulky. The alignment of fibers to the microgripper waveguide requires use of $2 \mathrm{XYZ}$ stages just to hold the fibers in place. This makes the device very un-portable. The aim is to use the device as a plug and play device that can be assembled in a few minutes. The first step towards that aim has been taken already with a new mask design which has slots 
for input and output fibers. This would enable quick assembly of the microgripper. Other major issue is the piezo electric actuation mechanism. The device cannot be used in many biological applications because of the sterilization issue. Moreover the piezo element is so big in size that the input and output fibers have to be bend in order to connect to the waveguides which leads to losses. An internal actuation mechanism would help solve these issues. Also it was seen from the imaging results that the illumination across the microgripper facet is not uniform. The imaging of a multimode fiber facet was also done and the non-uniform illumination of facet was observed here too. But the interesting part was the modes were reproducible to some extent. A series of pictures were taken of multimode fibers facet with fibers in initial position, then the fiber was messed and again it was let go to initial position. Though not $100 \%$ same but the difference between first and third image was minimal. A system can be designed with micrometer accuracy such that the modes can be exactly reproducible. Fluorescence detection is one other thing that needs to be improved also. From the experiments, it was evident that the fluorescence was escaping through the sidewalls of the SU8 films rather than coming out of the surface of the films. This means that the light guides inside SU8 very effectively and undergoes total internal reflection. It would be interesting to make very small patterned structures out of the quantum dot tagged SU8 films such that the light comes out through the surface of the films instead of guiding and coming out of the edges. This would help in inserting the films inside the microgripper gap and then detecting the fluorescence. 


\section{REFERENCES}

[1] Design Optimization for an Electro-Thermally Actuated Polymeric Microgripper, R. Voicu, R. Muller, L. Eftime, EDA Publishing/DTIP 2008

[2] N.-T. Nguyen, S.-S. Ho, and C. Lee-Ngo Low, " A polymeric microgripper with integrated thermal actuators", J. Micromech. Microeng. 14 (2004) 969-974.

[3] I. Roch et al, "Fabrication and characterisation of an SU-8 gripper actuated by a shape memory alloy thin film”, $2003 \mathrm{~J}$. Micromech. Microeng., 13, 330-336

[4] H. Lorenz, M. Despont, M. Fahrni, N. LaBianca, P. Vettiger, and P. Renaud. SU-8: a low-cost negative resist for MEMS. J. Micromech. Microeng 7(1997), 121-124

[5] L. Dellmann, S. Roth, C. Beuret, G. Racine, H. Lorenz, M. Despont, P. Renaud, P. Vettiger, and N. de Rooij. Fabrication process of high aspect ratio elastic structures for piezoelectric motor applications. in Proc. Transducers 1997, Chicago, (1997), 641-644

[6] G. Voskerician, M. S. Shive, R. S. Shawgo, H. von Recum, J. M. Anderson, M. J. Cima, and R. Langer. "Biocompatibility and biofouling of MEMS drug delivery devices", Biomaterials, vol. 24, pp. 1959-1967, 2003

[7] N. Chronis, L. P. Lee, "Electrothermally activated SU-8 microgripper for single cell manipulation in solution", Journal of Microelectromechanical Systems (2005)

[8] Belen Solano, David Wood, "Design and testing of a polymeric microgripper for cell manipulation", Microelectronic Engineering 84 (2007) 1219-1222

[9] M. Carrozza et al, "Towards a force-controlled microgripper for assembling biomedical microdevices", J. Micromech. Microeng. 10 (2000) 271-276

[10] C. Kim, A. Pisano, R. Muller, "Silicon-processed overhanging microgripper", J. MEMS 1 (1992).

[11] B.E. Volland, H. Heerlein, I.W. Rangelow, "Electrostatically driven microgripper", Microelectron. Eng. 61 (2002) 1015-1023.

[12] R. Wierzbicki, K. Houston, H. Heerlein, W. Barth, T. Debski, A. Eisinberg, A. Mendicassi, M.C. Carrozza, P. Dario, "Design and fabrication of an electrostatically driven microgripper for blood vessel manipulation", Microelectron. Eng. 83 (2006) 1651-1654.

[13] H. Du, C. Su, M. Lim, W. Jin, Smart Mater, "A micromachined thermally-driven gripper: a numerical and experimental study", Struct. 8 (1999) 616-622.

[14] K. Ivanova et al, "Thermally driven microgripper as a tool for micro assembly", Microelectron. Eng. 83 (2006) 1393-1395. 
[15] F. Beyeler, A. Neild, S. Oberti, D. J. Bell, Yu Sun, J. Dual, and B. J. Nelson, "Monolithically Fabricated Microgripper With Integrated Force Sensor for Manipulating Microobjects and Biological Cells Aligned in an Ultrasonic Field," IEEE Journal of Microelectromechanical Systems, vol. 16, p. 8, February 2007.

[16] J. Park and W. Moon, "A hybrid-type micro-gripper with an integrated force sensor," Microsystem Technologies, vol. 9, p. 7, October 2003.

[17] M. S.-C. Lu, Z.-H. Wu, C.-E. Huang, S.-J. Hung, M.-H. Chen, and Y.-C. King, "CMOS micromachined grippers with on-chip optical detection," Journal of Micromechanics and Microengineering, vol. 17, p. 7, February 6, 2007.

[18] Jose A. Martinez, "A Micro-Opto-Electro- Mechanical System (MOEMS) for Microstructure Manipulation" (PhD dissertation, Florida International University, 2008.)

[19] Optical fiber tutorial (Optic fiber-Communication fiber), Fiber Optics for sale Co.

[20] M. Young, Optics and Lasers: Including Fibers and Optical Waveguide, 4 ed. Berlin; New York: Springer-Verlag, 1993.

[21] J. C. Palais, Fiber optic comunications, Fifth ed. New Jersey: Pearson Education, Inc., 2005.

[22] S. O. Kasap, Optoelectronic devices and photonics: principles and practices. New Jersey: Prentice-Hall, Inc., 2001.

[23] J. A. Martinez, T. Liu, and R. R. Panepucci, "Micro-opto-electro-mechanical system (MOEMS) for microstructure manipulation and optical characterization," in SPIE 2007. San diego, California, 2007, p. 9.

[24] Tao Liu, Jose A. Martinez, Amit Bhanushali, and Roberto R. Panepucci, "Waveguide Microgripper Power Distribution", Integrated Photonics and Nanophotonics Research and Applications (IPNRA) Boston, Massachusetts July 13, 2008

[25] Modal Noise in Multimode Fibers under Restricted I aunch Conditions George C. Papen and G. Matthew Murphy]

[26] "Radiation damage of Cadmium Selenide nanocrystals", Vanderbilt Materials Physics group 